\title{
Chaotic properties of dilute two- and three-dimensional random Lorentz gases: Equilibrium systems
}

\author{
H. van Beijeren \\ Institute for Theoretical Physics, University of Utrecht, Postbus 80006, Utrecht 3508 TA, The Netherlands \\ Arnulf Latz and J. R. Dorfman \\ Department of Physics, Institute for Physical Science and Technology, University of Maryland, College Park, Maryland 20742
}

(Received 17 October 1997)

\begin{abstract}
We compute the Lyapunov spectrum and the Kolmogorov-Sinai entropy for a moving particle placed in a dilute, random array of hard-disk or hard-sphere scatterers, i.e., the dilute Lorentz gas model. This is carried out in two ways. First we use simple kinetic theory arguments to compute the Lyapunov spectrum for both two- and three-dimensional systems. In order to provide a method that can easily be generalized to nonuniform systems we then use a method based upon extensions of the Lorentz-Boltzmann (LB) equation to include variables that characterize the chaotic behavior of the system. The extended LB equations depend upon the number of dimensions and on whether one is computing positive or negative Lyapunov exponents. In the latter case the extended LB equation is closely related to an "anti-Lorentz-Boltzmann equation" where the collision operator has the opposite sign to the ordinary LB equation. Finally, we compare our results with computer simulations of Dellago and Posch [Phys. Rev. E 52, 2401 (1995); Phys. Rev. Lett. 78, 211 (1997)] and find very good agreement. [S1063-651X(98)05204-0]

PACS number(s): 05.45. $+\mathrm{b}$
\end{abstract}

\section{INTRODUCTION}

The Lorentz model of a gas of noninteracting particles that collide with fixed scatterers has been a basic model for acquiring an understanding of fundamental issues in both the kinetic theory of gases and the general theory of nonequilibrium phenomena in fluids and solids [1,2]. In this paper we use the Lorentz gas to study important features of the chaotic behavior of systems that show typical equilibrium and nonequilibrium behavior such as the existence of a spatially homogeneous equilibrium state and normal diffusion of moving particles among the scatterers. Since the particles do not collide with each other the Lorentz gas can readily be analyzed in terms of the properties of one moving particle in the set of fixed scatterers. Here we consider the scatterers to be placed at random in space, subject only to the condition that they are not allowed to overlap with each other. The chaotic properties of a particle moving in a periodic array of nonoverlapping hard disks have been studied extensively, especially for the case where the density of scatterers is sufficiently high that the moving particle is unable to travel unimpeded through the lattice (the case of finite horizon) [3]. It is known that under appropriate mathematical conditions the random Lorentz gas is a $K$ system [4] and that the periodic Lorentz gas with finite horizon is a Bernoulli system $[5,6]$. These results are sufficient to prove that the gas has a well-defined equilibrium state and that suitably defined initial ensemble distributions will approach equilibrium distributions for long enough times. However, for the random case there are very few analytic results for quantities that characterize the chaotic behavior of the moving particle. There is a conjecture by Krylov that the positive Lyapunov exponents for the moving particle are proportional to $n a^{d-1} v \ln \left[n a^{d}\right]^{-1}$ if $n a^{d} \ll 1$, where $n=N / V$ is the number density of $N$ scatterers of ra- dius $a$ in volume $V$ and $v$ is the constant speed of the moving particle [7]. This conjecture has been verified by Chernov, who argued that for low enough densities the periodic and the random Lorentz gas should have the same value of the Kolmogorov-Sinai entropy and then calculated this quantity for a periodic system at low density. Chernov obtained the results [8]

$$
\begin{gathered}
h_{\mathrm{KS}}=\lambda^{+} \simeq-2 n a v \ln n a^{2} \text { for } d=2, \\
h_{\mathrm{KS}}=\lambda_{1}^{+}+\lambda_{2}^{+} \simeq-2 \pi n a^{2} v \ln n a^{3} \text { for } d=3 .
\end{gathered}
$$

Here $\lambda^{+}$denotes a positive Lyapunov exponent. Simple considerations of the number of degrees of freedom and the conservation of energy show that for a two-dimensional Lorentz gas there can be no more than one positive Lyapunov exponent and for a three-dimensional gas there can be at most two of them. The quantity $h_{\mathrm{KS}}$ is the Kolmogorov-Sinai (KS) entropy, which for a closed, isolated ergodic system, such as the one considered by Chernov, is equal to the sum of the positive Lyapunov exponents, according to Pesin's theorem [9]. Chernov's results are only the first terms in the density expansion of $h_{\mathrm{KS}}$ for a random Lorentz gas and up until the present work no further analytic results had been known for either the density-dependent corrections to these results or, for three-dimensional systems, the individual Lyapunov exponents for the random gas. Recently, Dahlqvist has been able to calculate the density corrections for periodic Lorentz gas where small hard-disk scatterers are placed at the vertices of a square lattice [10].

We have been able to use familiar methods from the kinetic theory of gases to calculate the Lyapunov spectrum and the KS entropy for random Lorentz gases at low densities [11-14]. We can do this for closed, isolated systems as well as for closed systems in a magnetic field, open systems (with 
escape of particles), and systems where the moving particle is charged and subjected to an electric field plus a thermostat that maintains a constant kinetic energy even in the presence of the electric field. These two latter cases are of particular interest because of their importance for methods that relate dynamical quantities such as Lyapunov exponents and KS entropies to transport coefficients, in this case the diffusion coefficient for the moving particle $[15,16]$. This paper will be devoted to obtaining the low-density results for Lyapunov exponents and KS entropies under the various situations mentioned above. Extensions of these results to higher densities and to other quantities will be presented elsewhere.

In this paper we shall consider dilute, equilibrium Lorentz gases in two or three dimensions, consisting, respectively, of randomly placed but nonoverlapping, fixed hard-disk or hard-sphere scatterers and a point particle of mass $m$ and speed $v$ moving among them. The collisions with the scatterers are taken to be elastic. In the future we plan to generalize this model to nonequilibrium situations with thermostatted electric fields, and/or open systems with absorbing boundaries. The plan of this paper is as follows. In Sec. II we present an elementary kinetic theory argument that correctly provides the low-density values of the Lyapunov exponents and KS entropy of the Lorentz gas in two and three dimensions for closed systems and without external fields. In Sec. III we consider a more formal approach to these quantities due to Sinai [3], which will form the basis of the extension of the kinetic theory approach to nonuniform systems. There we provide the fundamental geometric formulas of Sinai, which relate the Lyapunov exponents and KS entropy of a Lorentz gas to the properties of a radius of curvature matrix. Then we use the ergodic properties of the moving particle to express the Lyapunov exponents and KS entropy in terms of averages over an equilibrium ensemble. In Sec. IV we show that the pertinent distribution functions can be obtained from the solution of an appropriate extended Lorentz-Boltzmann equation and in Sec. V we calculate the KS entropy of the two- and three-dimensional Lorentz gases at low densities. In Sec. VI we consider the negative Lyapunov exponents and show how they can be obtained from a solution of an "antiLorentz-Boltzmann" equation. This will be important for the extension of the theory to treat thermostated systems. We conclude in Sec. VII with a comparison of these results with the results of computer simulations by Posch and Dellago [17] and with a discussion of the applications of the methods developed here to more general systems.

\section{VELOCITY DEVIATION METHOD FOR LYAPUNOV EXPONENTS AND KS ENTROPIES}

We consider a system of $N d$-dimensional hard-sphere scatterers placed randomly in space in a $d$-dimensional volume $V$ at low density. Here $d=2,3$, the spheres have a radius $a$, and the number density of the spheres $n=N / V$ satisfies $n a^{d} \ll 1$. The moving particle travels freely between elastic collisions with the scatterers. The phase point $x$ of the particle, i.e., its position and velocity, $x=(\vec{r}, \vec{v})$ satisfies the equations of motion $\dot{\vec{r}}=\vec{v}$ and $\dot{\vec{v}}=0$ between collisions. At a collision of the moving particle with a scatterer, the velocity of the moving particle changes according to

$$
\vec{v}^{+}=\vec{v}-2(\vec{v} \cdot \hat{n}) \hat{n},
$$

where $\vec{v}^{+}$is the velocity after collision and $\hat{n}$ is the unit vector in the direction from the center of the scatterer to the point of impact of the moving particle at collision [18]. This completely specifies the dynamics of the particle given its initial phase and the locations of all of the scatterers.

The Lyapunov exponents characterize the rate of exponential separation or of exponential convergence of infinitesimally nearby trajectories on the $(2 d-1)$-dimensional constant energy surface $\mathcal{E}$ [9]. Since the Lorentz gas is a symplectic, Hamiltonian system, if there are nonzero Lyapunov exponents they come in pairs of positive and negative values $\pm \lambda_{i}[19]$. However, the Lyapunov exponent for displacements in the direction of the trajectory is zero since two phase points on the same physical trajectory will follow one another without exponential separation or contraction. Therefore, there can be at most $d-1$ positive and $d-1$ negative Lyapunov exponents for our system.

To treat the Lyapunov exponents we consider a bundle of infinitesimally nearby trajectories on $\mathcal{E}$ and follow the motion of this bundle in time. If the phase point on one reference trajectory in this bundle is given by $x(t)$ we denote the deviation of another trajectory in the bundle from $x(t)$ by $\delta x(t)=(\delta \vec{r}(t), \delta \vec{v}(t))$. Equations of motion for $\delta x(t)$ follow immediately from the equations of motion for $x(t)$. Since the moving particle has only kinetic energy the requirement that both trajectories lie on the same energy surface immediately leads to the property $\vec{v}(t) \cdot \delta \vec{v}(t)=0$ for all $t$. Without loss of generality, we may replace $\delta \vec{r}(t)$ by the vector of closest approach between the two trajectories, i.e. we set $\delta \vec{r}(t) \cdot \vec{v}(t)=0$. [From here on we will use the notation $\delta \vec{r}(t)$ for this vector of closest approach of the perturbed trajectory to $\vec{r}(t)$. Notice that if $\delta \vec{r}(t) \cdot \vec{v}(t)=0$ at $t=0$ it remains so at all later times, by virtue of Eqs. (3)-(6).] In between collisions the spatial and velocity deviations change with time according to

$$
\begin{gathered}
\delta \dot{\vec{r}}=\delta \vec{v}, \\
\delta \dot{\vec{v}}=0 .
\end{gathered}
$$

The change of $\delta x(t)$ at collisions requires some analysis, which has been provided by Gaspard and Dorfman [20] and Dellago, Posch, and Hoover [21]. These authors have shown that the change in $\delta x$ at the instant of a collision is given by

$$
\begin{gathered}
\delta \vec{r}^{+}=[\mathbf{1}-2 \hat{n} \hat{n}] \cdot \delta \vec{r}, \\
\delta \vec{v}^{+}=[\mathbf{1}-2 \hat{n} \hat{n}] \cdot \delta \vec{v}+\frac{2}{a}\left[\vec{v} \hat{n}-\hat{n} \vec{v}+\frac{v^{2}}{(\vec{v} \cdot \hat{n})} \hat{n} \hat{n}-(\vec{v} \cdot \hat{n}) \mathbf{1}\right] \cdot \delta \vec{r},
\end{gathered}
$$

where $\vec{v}, \delta \vec{r}, \delta \vec{v}$ are the velocity of the moving particle, the spatial deviation, and the velocity deviation of the nearby trajectory, immediately before the collision with the scatterer, while the "+" variables denote the values immediately after collision. It is important to note here that the velocity deviation $\delta \vec{v}$ does not change between collisions but does undergo an instantaneous change at each collision with a scatterer. 
Suppose that we prepare a trajectory bundle with initial values of $\delta x(0)$ and we follow the motion $\delta x(t)$ in time. We can relate the largest positive Lyapunov exponent to the asymptotic growth of the ratio $|\delta \vec{v}(t)| /|\delta \vec{v}(0)|$ by

$$
\lambda_{\max }=\lim _{t \rightarrow \infty} \frac{1}{t} \ln \left[\frac{|\delta \vec{v}(t)|}{|\delta \vec{v}(0)|}\right] .
$$

This follows from the observation that if there are positive Lyapunov exponents, then two infinitesimally close trajectories will eventually separate in time unless they are so precisely arranged that they approach each other exponentially in time. However, the latter situation occurs only on sets of zero measure (the stable manifold) in tangent space. Furthermore, this exponential separation occurs both in configuration space and in velocity space with the same exponential factor since velocities and positions are related by a simple time derivative that does not affect the exponential separation rate and we may consider the separation in velocity space alone. In Sec. III we consider another calculation of the Lyapunov exponents, which treats the separation in configuration space, and we obtain the same results.

Similarly, the sum of all of the positive Lyapunov exponents can be obtained by following the growth of a volume element in velocity space as

$$
\sum_{\lambda_{i}>0} \lambda_{i}=\lim _{t \rightarrow \infty} \frac{1}{t} \ln |\operatorname{det} \mathbf{A}(t)|
$$

with $\mathbf{A}(t)$ describing the linear relationship between $\delta \vec{v}(t)$ and $\delta \vec{v}(0)$, i.e.,

$$
\delta \vec{v}(t)=\mathbf{A}(t) \cdot \delta \vec{v}(0) .
$$

This result follows because the time evolution of the vector $\delta \vec{v}$, which has $d-1$ independent components, is dominated by the $d-1$ largest eigenvalues and corresponding eigenvectors of the matrix $\mathbf{A}(t)$, which are precisely the positive eigenvalues. Suppose now that the moving particle undergoes a series of $\mathcal{N}$ collisions in the time interval $[0, t]$ with scatterers, which we label $s_{1}, s_{2}, \ldots, s_{\mathcal{N}}$. Since the width of the trajectory bundle is infinitesimal, each trajectory within it has the same number of collisions with each scatterer in the same time. Since $\delta \vec{v}$, as noted before, changes only at collisions, one has

$$
\frac{|\delta \vec{v}(t)|}{|\delta \vec{v}(0)|}=\frac{\left|\delta \vec{v}_{\mathcal{N}}^{+}\right|}{\left|\delta \vec{v}_{\mathcal{N}-1}^{+}\right|} \frac{\left|\delta \vec{v}_{\mathcal{N}-1}^{+}\right|}{\left|\delta \vec{v}_{\mathcal{N}-2}^{+}\right|} \cdots \frac{\left|\delta \vec{v}_{1}^{+}\right|}{\left|\delta \vec{v}_{0}\right|}
$$

where $\delta \vec{v}_{i}^{+}$is the velocity deviation immediately after the collision with scatterer $s_{i}$. For the same reason $\mathbf{A}(t)$ can change with time only at the instants of the collisions of the moving particle with the scatterer, so that

$$
\delta \vec{v}(t)=\delta \vec{v}_{\mathcal{N}}^{+}=\mathbf{a}_{\mathcal{N}} \cdot \delta \vec{v}_{\mathcal{N}-1}^{+}=\mathbf{a}_{\mathcal{N}} \cdot \mathbf{a}_{\mathcal{N}-1} \cdots \mathbf{a}_{1} \cdot \delta \vec{v}(0) .
$$

Here $\mathbf{a}_{j}$ is a matrix, to be defined below for the case where the density of scatterers is low, that expresses the change in the velocity deviation when the moving particle collides with scatterer $s_{j}$. Consequently,

$$
\operatorname{det} \mathbf{A}(t)=\prod_{i}^{\mathcal{N}} \operatorname{det} \mathbf{a}_{i}
$$

Of course the number of collisions $\mathcal{N}$ in time $t$ will depend upon $t$ and the initial value of the phase point $x(0)$. Expressions for the largest Lyapunov exponent and for the sum of positive Lyapunov exponents can be obtained from Eqs. (8)(13) as

$$
\begin{gathered}
\lambda_{\max }=\lim _{t \rightarrow \infty} \frac{\mathcal{N}}{t} \frac{1}{\mathcal{N}} \sum_{1}^{\mathcal{N}} \ln \frac{\left|\delta \vec{v}_{i}^{+}\right|}{\left|\delta \vec{v}_{i-1}^{+}\right|}, \\
\sum_{\lambda_{i}>0} \lambda_{i}=\lim _{t \rightarrow \infty} \frac{\mathcal{N}}{t} \frac{1}{\mathcal{N}} \sum_{1}^{\mathcal{N}} \ln \left|\operatorname{det} \mathbf{a}_{i}\right| .
\end{gathered}
$$

To proceed further we need to use the fact that the density of the scatterers is small, that is, $n a^{d} \ll 1$. This will allow us to determine the mean of the quantities appearing in the sums in Eqs. (14) and (15). Referring to Eq. (7), we note that $\delta \vec{r}$ appearing on the right-hand side is the spatial deviation of the moving particle just before a collision with a scatterer. Let us suppose that we consider the collision with scatterer $s_{i}$. Then immediately before this collision $\delta \vec{r}_{i}^{-}=\delta \vec{r}_{i-1}^{+}$ $+\tau_{i} \delta \vec{v}_{i-1}^{+}$, where $\delta x_{i-1}^{+}$denotes the spatial and velocity deviations just after the collision with the previous scatterer $s_{i-1}$ and $\tau_{i}$ is the time between the collision with scatterer $s_{i-1}$ and scatterer $s_{i}$. At low scatterer densities the time between collisions will typically be inversely proportional to the density of scatterers, so that the ratio of the order of magnitudes of the first to the second term in the above expression for $\delta \vec{r}_{i}^{-}$will approach zero as the scatterer density decreases. Therefore, to leading order in the density, $\delta \vec{r}_{i}^{-}$ $=\tau_{i} \delta \vec{v}_{i-1}^{+}$. We then obtain a low-density value for $\delta \vec{v}_{i}^{+}$ given by

$$
\begin{aligned}
\delta \vec{v}_{i}^{+}= & {\left[(\mathbf{1}-2 \hat{n} \hat{n})+\frac{2 \tau_{i}}{a}\left(\vec{v}_{i-1}^{+} \hat{n}-\hat{n} \vec{v}_{i-1}^{+}-\left(\vec{v}_{i-1}^{+} \cdot \hat{n}\right) \mathbf{1}\right.\right.} \\
& \left.\left.+\frac{v^{2}}{\left(\vec{v}_{i-1}^{+} \cdot \hat{n}\right)} \hat{n} \hat{n}\right)\right] \cdot \delta \vec{v}_{i-1}^{+} \\
\equiv & \mathbf{a}_{i} \cdot \delta \vec{v}_{i-1}^{+} .
\end{aligned}
$$

Now we have expressions for the change in the velocity deviation at collision and for the matrix $\mathbf{a}$, both of which are needed for the calculations outlined above. To evaluate the sums appearing in Eqs. (14) and (15), we note that at low densities none of the collisions are correlated with any previous collision, that is, the leading contribution to the Lyapunov exponents come from collision sequences where the moving particle does not encounter the same scatterer more than once in the sequence. (In two dimensions the particle will hit the same scatterer an infinite number of times. However, the effects of such processes are of higher density and can be neglected here since the times between successive collisions with the same scatterer become typically very large as the density of scatterers approaches zero). Therefore, we can treat each term in the sums in Eqs. (14) and (15) as being independent of the other terms in the sum. We have expressed $\lambda_{\max }$ and the sum of the positive Lyapunov expo- 
nents as arithmetic averages, but for long times and with independently distributed terms in the average, we can replace the arithmetic averages by ensemble averages over a suitable equilibrium ensemble. That is

$$
\lambda_{\max }=\nu\left\langle\ln \left[\frac{\left|\delta \vec{v}^{+}\right|}{\left|\delta \vec{v}_{-}\right|}\right]\right\rangle
$$

and

$$
\sum_{\lambda_{i}>0} \lambda_{i}=\nu\langle\ln |\operatorname{det} \mathbf{a}|\rangle
$$

where $\nu$ is the (low-density) value of the collision frequency $\mathcal{N} / t$ as $t$ becomes large and the angular brackets denote an equilibrium average.

We now consider a typical collision of the moving particle with one of the scatterers. The free time between one collision and the next is sampled from the normalized equilibrium distribution of free times [18] $P(\tau)$ given at low densities by

$$
P(\tau)=\nu e^{-\nu \tau}
$$

The construction of the matrix a requires some geometry and depends on the number of dimensions of the system. In any

$$
\widetilde{\mathbf{a}}=\left(\begin{array}{c}
1+\Lambda\left(\cos ^{2} \phi+\sin ^{2} \phi \cos ^{2} \alpha\right) \\
\Lambda \sin ^{2} \phi \cos \alpha \sin \alpha \\
0
\end{array}\right.
$$

One easily finds

$$
\frac{\left|\delta \vec{v}^{+}\right|}{\left|\delta \vec{v}^{-}\right|}=\frac{2 \tau v}{a}\left[\frac{\cos ^{2}(\alpha-\psi)}{\cos ^{2} \phi}+\sin ^{2}(\alpha-\psi) \cos ^{2} \phi\right]^{1 / 2}
$$

and

$$
\operatorname{det} \widetilde{\mathbf{a}}=\operatorname{det} \mathbf{a}=\left(\frac{2 v \tau}{a}\right)^{2}
$$

to leading order in $v \tau / a$.

To complete the calculation we must evaluate the averages appearing in Eqs. (17) and (18). That is, we average over the distribution of free times and over the rate at which scattering events are taking place with the various scattering angles. Additionally, in three dimensions an average over a stationary distribution of angles $\psi$ has to be performed in general. Due to the isotropy of the scattering geometry $\psi$ can here be absorbed in a redefinition $\alpha^{\prime}=\alpha-\psi$ of the azimuthal angle $\alpha$. This will not be true anymore if the isotropy of velocity space is broken (e.g., by an external field). The appropriate average of a quantity $F$ takes the simple form case we take the velocity vector before collision $\vec{v}$ to be directed along the $z$ axis and take $\hat{n} \cdot \vec{v}=-v \cos \phi$. The velocity deviation before collision $\delta \vec{v}^{-}$is perpendicular to the $z$ axis. Then it is a simple matter to compute $\left|\delta \vec{v}^{+}\right| /\left|\delta \vec{v}^{-}\right|$ and $\mid$ deta $\mid$. For two-dimensional systems $\delta \vec{v}$ and the matrix a are given in this representation by

$$
\delta \vec{v}^{-}=\left(\begin{array}{l}
1 \\
0
\end{array}\right)\left|\delta \vec{v}^{-}\right|, \quad \mathbf{a}=\left(\begin{array}{cc}
(1+\Lambda) \cos 2 \phi & \sin 2 \phi \\
(1+\Lambda) \sin 2 \phi & -\cos 2 \phi
\end{array}\right),
$$

where we introduced $\Lambda=2 v \tau / a \cos \phi$, where $-\pi / 2 \leqslant \phi$ $\leqslant \pi / 2$. To leading order in $v \tau / a$ we find that

$$
\frac{\left|\delta \vec{v}^{+}\right|}{\left|\delta \vec{v}^{-}\right|}=\Lambda, \quad|\operatorname{det} \mathbf{a}|=\Lambda
$$

For three-dimensional systems the unit vector $\hat{n}$ can be represented as $\hat{n}=-(\cos \phi) \hat{z}+\sin \phi(\cos \alpha) \hat{x}+\sin \phi(\sin \alpha) \hat{y}$. Now the ranges of the angles $\phi$ and $\alpha$ are $0 \leqslant \phi \leqslant \pi / 2$ and $0 \leqslant \alpha \leqslant 2 \pi$. There is an additional angle $\psi$ in the $(x, y)$ plane such that the velocity deviation before collision $\delta \vec{v}^{-}$ $=\left|\delta \vec{v}^{-}\right|[\hat{x} \cos \psi+\hat{y} \sin \psi]$. It is somewhat more convenient to use a symmetric matrix $\widetilde{\mathbf{a}}=(\mathbf{1}-\mathbf{2} \hat{\mathbf{n}} \hat{\mathbf{n}}) \cdot \mathbf{a}$ given by

$$
\left.\begin{array}{cc}
\Lambda \sin ^{2} \phi \cos \alpha \sin \alpha & 0 \\
1+\Lambda\left(\cos ^{2} \phi+\sin ^{2} \phi \sin ^{2} \alpha\right) & 0 \\
0 & 1
\end{array}\right)
$$

$$
\langle F\rangle=\frac{1}{J} \int_{0}^{\infty} d \tau \int d \hat{n}(\cos \phi) P(\tau) F
$$

where $P(\tau)$ is the free time distribution given by Eq. (19) and $J$ is a normalization factor obtained by setting $F=1$ in the numerator. The integration over the unit vector $\hat{n}$, i.e., over the appropriate solid angle, ranges over $-\pi / 2 \leqslant \phi$ $\leqslant \pi / 2$ in two dimensions and over $0 \leqslant \phi \leqslant \pi / 2$ and $0 \leqslant \alpha$ $\leqslant 2 \pi$ in three dimensions. After carrying out the required integrations we find that

$$
\lambda^{+}=\lambda_{\max }=2 n a v\left[-\ln \left(2 n a^{2}\right)+1-\mathcal{C}\right]+\cdots
$$

for two dimensions. Here $\mathcal{C}$ is Euler's constant and the terms not given explicitly in Eq. (26) are higher order in the density. Similarly, for the three-dimensional Lorentz gas we obtain

$$
\begin{gathered}
\lambda_{\max }^{+}=n a^{2} v \pi\left[-\ln (\tilde{n} / 2)+\ln 2-\frac{1}{2}-\mathcal{C}\right]+\cdots, \\
\lambda_{\max }^{+}+\lambda_{\min }^{+}=2 n a^{2} v \pi[-\ln (\tilde{n} / 2)-\mathcal{C}]+\cdots,
\end{gathered}
$$

from which it follows that

$$
\lambda_{\min }^{+}=n a^{2} v \pi\left[-\ln (\tilde{n} / 2)-\ln 2+\frac{1}{2}-\mathcal{C}\right]+\cdots,
$$


where $\tilde{n}=n a^{3} \pi$. We have therefore determined the Lyapunov spectrum for the equilibrium Lorentz gas at low densities in both two and three dimensions [11,13]. We note that the two positive Lyapunov exponents for three dimensions differ slightly and that we were able to get individual values because we could calculate the largest exponent and the sum of the two exponents. We could not determine all of the Lyapunov exponents for a $(d>3)$-dimensional Lorentz gas this way. Moreover, for a spatially inhomogeneous system, such as those considered in the application of escaperate methods, the simple kinetic arguments used here are not sufficient and Boltzmann-type methods are essential for the determination of the Lyapunov exponents and KS entropies. We will comment further on these results in Sec. VII after we have obtained them again by a more formal method based upon the radius of curvature matrix method of Sinai [3].

\section{THE RADIUS OF CURVATURE MATRIX}

Our analysis in this section is based upon the geometric arguments given by Sinai [3] for the relationships between the Lyapunov exponents, the KS entropy, and the radius of curvature matrix, which describes the time evolution of the separation of nearby phase-space trajectories of the moving particle. Unlike the method presented in the preceding section, this method treats the time evolution of the spatial separation of a bundle of trajectories rather than the evolution of the velocity separation of the bundle. Here we summarize these considerations, referring the reader to the literature for further details $[3,20]$.

As before, the trajectory of the moving particle is specified by the phase $x(t)=(\vec{r}(t), \vec{v}(t))$ and we take a $(2 d-2)$-dimensional plane $\Sigma,\left(\delta \vec{r}_{\perp}(t), \delta \vec{v}_{\perp}(t)\right)$, through $x(t)$, where both $\delta \vec{r}_{\perp}(t)$ and $\delta \vec{v}_{\perp}(t)$ are perpendicular to the velocity $\vec{v}(t)$. The nearby trajectories will intersect $\Sigma$ and we measure the separation of the trajectories by vectors of dimension $2(d-1)$ in $\Sigma, \delta x_{\perp}(t)$, given as

$$
\delta x_{\perp}(t)=\left(\begin{array}{c}
\delta \vec{r}_{\perp}(t) \\
\delta \vec{v}_{\perp}(t)
\end{array}\right) .
$$

The time development of $\delta x_{\perp}$ is given in terms of a monodromy matrix $\mathbf{M}\left(t, t_{0}\right)$ satisfying

$$
\delta x_{\perp}(t)=\mathbf{M}\left(t, t_{0}\right) \cdot \delta x_{\perp}\left(t_{0}\right) .
$$

The matrix $\mathbf{M}\left(t, t_{0}\right)$ follows the motion of the particle. It changes continuously with time $t$ in the intervals between collisions and undergoes a discontinuous change at the instants of collisions of the particle with the scatterers. Between collisions the monodromy matrix has the form

$$
\mathbf{M}\left(t, t_{0}\right)_{\text {free flight }}=\left(\begin{array}{cc}
\mathbf{1} & \left(t-t_{0}\right) \mathbf{1} \\
\mathbf{0} & \mathbf{1}
\end{array}\right) .
$$

At the instant of a collision there is a discontinuous rotation of the velocity of the moving particle from its value before collision $\vec{v}$ to its value after collision $\vec{v}^{+}$ $=\vec{v}-2 \hat{n}(\vec{v} \cdot \hat{n})$ as before. Since the velocity of the particle changes discontinuously at collision, the plane $\Sigma$ also rotates and the components of the displacement vector $\delta x_{\perp}$ change instanta- neously. The changes in the components of $\delta \vec{r}_{\perp}$ and $\delta \vec{v}_{\perp}$ at the instant of collision are given in Eqs. (6) and (7) above.

In order to determine the Lyapunov exponents for this system we need to examine the rate of separation or of approach of infinitesimally close trajectories. This can now be done with the aid of the radius of curvature operators $\boldsymbol{\rho}_{u}$ and $\boldsymbol{\rho}_{s}$, acting on a $(d-1)$-dimensional space of velocity deviation vectors orthogonal to $\vec{v}$. Here the subscripts $u$ and $s$ denote operators describing unstable, or expanding, and stable, or contracting, trajectory bundles, respectively. The operator $\boldsymbol{\rho}_{u}$ is defined by the relation that

$$
\delta \vec{r}_{\perp}(t)=\frac{1}{v} \boldsymbol{\rho}_{u}(t) \cdot \delta \vec{v}_{\perp}(t),
$$

together with the conditions $\boldsymbol{\rho}_{u} \cdot \vec{v}=\vec{v} \cdot \boldsymbol{\rho}_{u}=0$. This definition is motivated by the observation that if the velocity deviation $\delta \vec{v}$ describes separating trajectories, then we can apply ray optics to describe the separation of the trajectories [3]. In the transverse plane this separation will be given by an arclength equal to a radius of curvature multiplied by an infinitesimal initial angular separation. We have chosen the units in Eq. (33) so that the radius of curvature operator has the dimension of a length. The radius of curvature operator can be represented as a $(d-1) \times(d-1)$ matrix since both $\delta \vec{r}_{\perp}$ and $\delta \vec{v}_{\perp}$ are defined in a plane perpendicular to $\vec{v}$. Now suppose that we consider some initial velocity deviation $\delta \vec{v}_{\perp}(0)$ corresponding to a diverging pencil of rays and we want to obtain an equation of motion for the radius of curvature matrix $\boldsymbol{\rho}_{u}$. We use the fact that the motion of the particle consists of periods of free flight punctuated by instantaneous collisions with the fixed scatterers. We first consider the free flight motion. From the fact that in free flight $\delta \vec{r}_{\perp}(t)$ $=\delta \vec{r}_{\perp}(0)+t \delta \vec{v}_{\perp}(0)$ and $\delta \vec{v}_{\perp}(t)=\delta \vec{v}_{\perp}(0)$, we infer that during free flight over a time interval $t$ from some initial time $t=0$, with initial value $\boldsymbol{\rho}(0)$, the radius of curvature matrix changes with time according to

$$
\boldsymbol{\rho}_{u}(t)=\boldsymbol{\rho}_{u}(0)+v t \mathbf{1}_{\perp},
$$

where $\mathbf{1}_{\perp}=\mathbf{1}-\hat{v} \hat{v}$ with $\hat{v}$ a unit vector in the direction of $\vec{v}$. Next we use Eqs. (6) and (7) to obtain the relation between the radius of curvature operator immediately before a collision $\boldsymbol{\rho}_{u}^{(-)}$and its value immediately after a collision $\boldsymbol{\rho}_{u}^{(+)}$. This calculation, while straightforward, requires a careful analysis in order to obtain a correct expression for $\boldsymbol{\rho}$ as a $(d-1) \times(d-1)$ matrix. This analysis is presented in Appendix A. There we find that at a collision $\boldsymbol{\rho}$ changes according to

$$
\begin{aligned}
{\left[\boldsymbol{\rho}_{u}^{-1(+)}\right]=} & \mathbf{U}\left\{\left[\boldsymbol{\rho}_{u}^{-1(-)}\right]+\frac{2}{a}\left[\hat{v} \hat{n}+\hat{n} \hat{v}-\frac{1}{\hat{v} \cdot \hat{n}} \hat{n} \hat{n}\right.\right. \\
& -(\hat{v} \cdot \hat{n}) \mathbf{1}]\} \mathbf{U},
\end{aligned}
$$

where $\mathbf{U}$ is the reflection operator $\mathbf{1}-2 \hat{n} \hat{n}$. The inverse radii of curvature tensors $\left[\boldsymbol{\rho}_{u}^{-1(-)}\right]$ and $\left[\boldsymbol{\rho}_{u}^{-1(+)}\right]$ are defined on the subspaces orthogonal to $\hat{v}$ and $\hat{v}^{\prime}$, respectively, and are 
extended to tensors on full space by requiring that their left and right inner products with $\hat{v}$ and $\hat{v}^{\prime}$, respectively, are zero again.

The sum of the positive Lyapunov exponents is connected to the radius of curvature operator $[3,8]$. Consider the spatial separation of a bundle of trajectories after a sequence of $n$ collisions labeled $1,2, \ldots, n$ that take place at times $t_{1}, t_{2}, \ldots, t_{n}$,

$$
\begin{aligned}
\delta \vec{r}_{\perp}^{(-)}\left(t_{n}\right)= & {\left[\mathbf{1}_{\perp}+v \tau_{n, n-1} \boldsymbol{\rho}_{u}^{-1(+)}\left(t_{n-1}\right)\right] \cdot \mathbf{U}(n-1) } \\
& \times\left[\mathbf{1}_{\perp}+v \tau_{n-1, n-2} \boldsymbol{\rho}_{u}^{-1(+)}\left(t_{n-2}\right)\right] \cdot \mathbf{U}(n-2) \cdots \\
& \times\left[\mathbf{1}_{\perp}+v \tau_{1,0} \boldsymbol{\rho}_{u}^{-1}(0)\right] \cdot \delta \vec{r}_{\perp}(0)
\end{aligned}
$$

where the initial time has been set to $t=0$, with initial values indicated for the spatial deviation vector $\delta \vec{r}_{\perp}(0)$ and for the radius of curvature operator $\boldsymbol{\rho}_{u}(0)$. Also $\mathbf{U}(j)=\mathbf{1}-2 \hat{n}_{j} \hat{n}_{j}$ is the reflection operator at the $j$ th collision. It is important to note that $\operatorname{det} \mathbf{U}(j)=-1$. From Eq. (36) the sum of the postive Lyapunov exponents can be derived as $[3,8]$

$$
\sum_{\lambda_{i}>0} \lambda_{i}=\lim _{t \rightarrow \infty} \frac{v}{t} \int_{0}^{t} d \tau \operatorname{Tr} \boldsymbol{\rho}_{u}^{-1}(\tau) .
$$

Equation (37) is Sinai's formula for the KS entropy for a moving particle in a system of fixed hard-sphere scatterers $[3,8]$. By combining Eqs. (34) and (35) one may obtain a continued-fraction representation for $\left[\boldsymbol{\rho}_{u}^{-1}(t)\right]$ [3], which, for a fixed final phase point $x(t)$ and initial $\boldsymbol{\rho}_{u}(0)$, converges rapidly with increasing $t$. So far we have not used any properties of the arrangement of the scatterers, so this formula is still quite general. In the case that the system is ergodic the time average can be replaced by an ensemble average, taken with an appropriate ensemble distribution function, so we can express the sum of the positive Lyapunov exponents as

$$
\sum_{\lambda_{i}>0} \lambda_{i}=v\left\langle\operatorname{Tr} \boldsymbol{\rho}_{u}^{-1}\right\rangle,
$$

where the angular brackets denote an average over an appropriate stationary ensemble reached in the course of time from smooth initial distributions. In the case of interest here, this distribution will be an equilibrium distribution, but in the future we will need to consider more general steady-state distribution functions.

Before completing this section we wish to give a simple derivation of Eq. (37) that applies to a Lorentz gas with any reasonable interaction between the moving particle and the scatterers. We use the fact that having defined the radius of curvature matrix $\boldsymbol{\rho}_{u}$, we may write

$$
\frac{d \delta \vec{r}_{\perp}(t)}{d t}=\delta \vec{v}_{\perp}(t)=v\left[\boldsymbol{\rho}_{u}^{-1}(t)\right] \cdot \delta \vec{r}_{\perp}(t),
$$

with the solution

$$
\delta \vec{r}_{\perp}(t)=\mathcal{T} \exp v \int_{0}^{t} d \tau\left[\boldsymbol{\rho}_{u}(\tau)\right]^{-1} \cdot \delta \vec{r}_{\perp}(0) .
$$

Here $\mathcal{T}$ denotes a time-ordering operator. Using the method of differential forms or equivalent methods [22] we see that the growth of a volume element $\delta V_{r}(t)$ in configuration space is given by

$$
\frac{\delta V_{r}(t)}{\delta V_{r}(0)}=\exp \left(v \int_{0}^{t} d \tau \operatorname{Tr}\left[\boldsymbol{\rho}_{u}(\tau)\right]^{-1}\right) .
$$

This result leads immediately to Eq. (37) for the sum of Lyapunov exponents. Vattay has shown how to construct the inverse of the radius of curvature matrix for a general potential [23]. It is straightforward generalizing the results obtained here to other short-range interaction potentials and it may well be possible to treat some cases with long-range interactions between the scatterers and the light particle. For the case of hard disks or spheres considered here, we can use the fact that the radius of curvature matrices at different times commute with each other if the times involved are all within the same time interval between one collision of the moving particle and a scatterer and the next collision to write

$$
\delta \vec{r}_{\perp}(t)=\left[\prod_{J=0}^{n} \mathbf{R}\left(t_{j+1}, t_{j}\right)\right] \cdot \delta \vec{r}_{\perp}(0),
$$

where

$$
\mathbf{R}\left(t_{j+1}, t_{j}\right)=\exp \left[v \int_{t_{j}}^{t_{j+1}} d \tau\left[\boldsymbol{\rho}_{u}(\tau)\right]^{-1}\right]
$$

and the prime on the product denotes that the times are to be ordered so that the times decrease from left to right in the product. By using Eq. (34) and carrying out the required integrals, one can easily see that this expression is equivalent to Eq. (36). Moreover, one can express the sum of the positive Lyapunov exponents as

$$
\sum \lambda_{i}^{+}=\lim _{t \rightarrow \infty} \frac{\mathcal{N}(t)}{t} \frac{1}{\mathcal{N}(t)} \sum_{j} v \int_{t_{j}}^{t_{j+1}} d \tau \operatorname{Tr}\left[\boldsymbol{\rho}_{u}(\tau)\right]^{-1} .
$$

We will use these expressions in Sec. VI and in the Appendixes.

In Sec. IV we discuss the distribution function appearing in the above ensemble average for the case that the scatterers are distributed at random with very low density, i.e., the mean free path of the moving particle is very large compared to the radius of the scatterers. In order to obtain the individual Lyapunov exponents we have to find the eigenvalues of the operator that appears on the right-hand side of Eq. (36). This operator can be expressed as a product of ( $d$ $-1) \times(d-1)$ matrices, which describe the collisions of the moving particle with the scatterers and the free motion in between collisions. Again, if the system is at low density the product of matrices can be considered to be a product of randomly distributed matrices since the time between collisions and the collision parameters will be sampled from a random distribution, corresponding to the random placement of scatterers. In Appendix B we show how methods from the theory for eigenvalues of products of random matrices [24] can be used to obtain the largest Lyapunov exponent. At higher densities of scatterers correlations between collisions 
will arise and one will have to take into account these correlations when computing the eigenvalues of the product of matrices.

\section{THE EXTENDED LORENTZ-BOLTZMANN EQUATION}

In order to evaluate the ensemble average appearing on the right-hand side of Eq. (38) we need to construct an equilibrium or, more generally, a steady-state distribution function for the radius of curvature matrix. The physics of the problem suggests that a method based upon the LorentzBoltzmann equation is appropriate here. That is, we have a particle moving in a random array of scatterers making only binary collisions with the scatterers. The particle moves freely between collisions and at a collision both the velocity and the radius of curvature matrix change instantaneously. Methods are now well known [1,2,18,26,27] for obtaining a generalized Lorentz-Boltzmann equation for the timedependent space and velocity distribution function $f(\vec{r}, \vec{v}, t)$ for the moving particle as a function of the density of the scatterers, at least in the case that the scatterers are nonoverlapping. (The case of overlapping scatterers is complicated by the fact that regions may exist where the particle would be trapped for all time. In a transport problem these regions need to be treated carefully since particles trapped in them will not diffuse beyond the borders of the trap). In this case it is possible to obtain an equation for the moving particle that includes the effects of uncorrelated binary collisions of the particle with the scatterers, excluded-volume effects, and the effects of correlated collision sequences of the moving particle with the scatterers. To lowest order in the density of the scatterers, the distribution function $f(\vec{r}, \vec{v}, t)$ satisfies the Lorentz-Boltzmann (LB) equation [1,2,18,27]

$$
\begin{aligned}
\frac{\partial f}{\partial t}+ & \vec{v} \cdot \frac{\partial f}{\partial \vec{r}}+\dot{\vec{v}} \cdot \frac{\partial f}{\partial \vec{v}} \\
= & n a^{d-1} \int d \hat{n}|\vec{v} \cdot \hat{n}|[\Theta(\vec{v} \cdot \hat{n}) f(\vec{r}, \vec{v}-2(\vec{v} \cdot \hat{n}) \hat{n}, t) \\
& -\Theta(-\vec{v} \cdot \hat{n}) f(\vec{r}, \vec{v}, t)] .
\end{aligned}
$$

Here $\hat{n}$ is a unit vector in the direction from the center of a scatterer to the point of impact at a collision and $\Theta(x)$ denotes the unit step function. The right-hand side of the LB equation describes the change in $f$ due to collisions as the difference between the gain and the loss of particles with velocity $\vec{v}$ from "restituting", and "direct" collisions, respectively. Higher-order density corrections to the right-hand side of Eq. (45), comprising the generalized LB equation, can be obtained using the appropriate set of BogoliubovBorn-Green-Kirkwood-Yvon (BBGKY) hierarchy equations and cluster-expansion methods [26,27,25]. These density corrections have been studied in some detail and it is well known that the so-called ring collision sequences are responsible for both logarithmic terms in the density expansion of the diffusion coefficient of the moving particle [27] and the long-time tails in the velocity autocorrelation function of the moving particle [28].
Our purpose here is to extend the LB equation by including the radius of curvature matrix among the variables described by the distribution function for the moving particle. We will consider only the low-density version of the kinetic theory for this distribution function and leave the discussion of higher-order density corrections, including the effects of correlated collision sequences on the Lyapunov exponents, to the future. Thus we wish to determine an equation, valid at low densities, for an extended distribution function $F(\vec{r}, \vec{v}, \boldsymbol{\rho}, t)$, where we dropped the subscript $u$ on $\boldsymbol{\rho}$, and we relate the distribution functions $F$ and $f$ by

$$
f(\vec{r}, \vec{v}, t)=\int d \boldsymbol{\rho} F(\vec{r}, \vec{v}, \boldsymbol{\rho}, t) .
$$

Given the stationary solution of the extended LB equation for $F$, we can determine the sum of the Lyapunov exponents as

$$
\sum_{\lambda_{i}>0} \lambda_{i}=v \int d \vec{r} d \vec{v} d \boldsymbol{\rho}\left[\operatorname{Tr} \boldsymbol{\rho}^{-1}\right] F(\vec{r}, \vec{v}, \boldsymbol{\rho}),
$$

assuming that $F$ is properly normalized.

An extended LB equation for $F$ that reduces to the usual LB equation for $f$ upon integration over the radius of curvature matrix elements can be obtained by following the heuristic derivation of the $\mathrm{LB}$ equation and simply modifying it to include the additional variables. That is, we consider a large collection of moving particles in the random array of scatterers and ask for an equation for the probability that a moving particle has its values for $\vec{r}, \vec{v}, \boldsymbol{\rho}$ in the range $d \vec{r}, d \vec{v}, d \boldsymbol{\rho}$ about $\vec{r}, \vec{v}, \boldsymbol{\rho}, \quad$ all at time $t$, i.e., $F(\vec{r}, \vec{v}, \boldsymbol{\rho}, t) d \vec{r} d \vec{v} d \boldsymbol{\rho}$. This probability changes in time due to free motion of the particles and due to collisions. The change in $F$ due to the free motion of the particles in time $d t$ is

$$
\begin{gathered}
{\left[F\left(\vec{r}+\vec{v} d t, \vec{v}, \boldsymbol{\rho}+v d t \mathbf{1}_{\perp}, t+d t\right)-F(\vec{r}, \vec{v}, \boldsymbol{\rho}, t)\right] d \vec{r} d \vec{v} d \boldsymbol{\rho}} \\
\quad=\left[\frac{\partial F}{\partial t}+\vec{v} \cdot \frac{\partial F}{\partial \vec{r}}+v \sum_{i=1} \frac{\partial F}{\partial \rho_{i i}}\right] d \vec{r} d \vec{v} d \boldsymbol{\rho} d t
\end{gathered}
$$

We used Eq. (34) to treat the change in the radius of curvature matrix during free particle motion and we have assumed that there are no external forces acting on the system. Otherwise we would need to include terms accounting for the changes in velocity and in the radius of curvature matrix over a time interval $d t$ due to the external force. If there were no collisions taking place in the system, then the right-hand side of Eq. (48) would be zero. However, the collisions account for the fact that the number of particles at $\vec{r}$ $+\vec{v} d t, \vec{v}, \boldsymbol{\rho}+v d t \mathbf{1}_{\perp}$ at time $t+d t$ is not equal to the number of particles at $\vec{r}, \vec{v}, \boldsymbol{\rho}$ at time $t$. To account for the change in $F$ due to collisions we consider the restituting and direct collisions separately. The direct collisions result in a loss of the particles with $\vec{r}, \vec{v}, \boldsymbol{\rho}$ over the interval $d t$ due to collisions with scatterers. Elementary kinetic theory considerations $[18,26]$ show that this loss is 


$$
n a^{d-1} \int d \hat{n}|\vec{v} \cdot \hat{n}| \Theta(-\vec{v} \cdot \hat{n}) F(\vec{r}, \vec{v}, \boldsymbol{\rho}, t) d \vec{r} d \vec{v} d \boldsymbol{\rho} d t
$$

The restituting or gain term is found by considering those collisions taking place in the time interval $[t, t+d t]$ that produce particles with $\vec{r}, \vec{v}, \boldsymbol{\rho}$. Again, elementary kinetic theory considerations show that this gain is given by

$$
n a^{d-1} \int d \hat{n}|\vec{v} \cdot \hat{n}| \Theta(\vec{v} \cdot \hat{n})\left|\frac{\partial \boldsymbol{\rho}^{\prime}}{\partial \boldsymbol{\rho}}\right| F\left(\vec{v}^{\prime}, \vec{r}, \boldsymbol{\rho}^{\prime}, t\right) d \vec{r} d \vec{v} d \boldsymbol{\rho} d t,
$$

where $\vec{v} \cdot \hat{n} \geqslant 0$,

$$
\vec{v}^{\prime}=\vec{v}-2(\vec{v} \cdot \hat{n}) \hat{n},
$$

and the radius of curvature matrix $\boldsymbol{\rho}^{\prime}$ is a restituting value such that the radius of curvature matrix becomes $\boldsymbol{\rho}$ after collision. From Eq. (35) and the identity $\mathbf{U} \cdot \hat{v}=\hat{v}^{\prime}$ one obtains the relationship

$$
\boldsymbol{\rho}^{\prime-1}=\mathbf{U}\left\{\boldsymbol{\rho}^{-1}-\frac{2}{a}\left[-\hat{v} \hat{n}-\hat{n} \hat{v}+\frac{1}{\hat{v} \cdot \hat{n}} \hat{n} \hat{n}+(\hat{v} \cdot \hat{n}) \mathbf{1}\right]\right\} \mathbf{U},
$$

with $\hat{v} \cdot \hat{n} \geqslant 0$. For two-dimensional systems the radius of curvature matrix can be represented by a scalar (namely, its nonvanishing eigenvalue) and the restituting value $\rho^{\prime}$ is given by

$$
\frac{1}{\rho^{\prime}}=\frac{1}{\rho}-\frac{2}{a \cos \phi}
$$

where $\phi$ is the angle between $\hat{n}$ and $\hat{v}$ with $-\pi / 2 \leqslant \phi$ $\leqslant \pi / 2$. For three-dimensional systems the radius of curvature matrix can be represented by a $2 \times 2$ matrix by choosing the principal axes of the coordinate frame orthogonal to $\hat{v}$. Defining angles $\phi$ through $\cos \phi=\hat{n} \cdot \hat{v}$, with $0 \leqslant \phi \leqslant \pi / 2$ and $\alpha$ as the angle between the second coordinate axis and the plane through $\hat{n}$ and $\hat{v}$, and multiplying Eq. (52) from the left and the right by $\mathbf{U}$ one can rewrite this equation as

$$
\mathbf{U} \boldsymbol{\rho}^{\prime-1} \mathbf{U}=\boldsymbol{\rho}^{-1}-\frac{2 \cos \phi}{a}\left(\begin{array}{ccc}
0 & 0 & 0 \\
0 & & \\
& \mathbf{P}
\end{array}\right)
$$

with

$$
\mathbf{P}=\left(\begin{array}{cc}
1+\tan ^{2} \phi \cos ^{2} \alpha & \tan ^{2} \phi \sin \alpha \cos \alpha \\
\tan ^{2} \phi \sin \alpha \cos \alpha & 1+\tan ^{2} \phi \sin ^{2} \alpha
\end{array}\right)
$$

Here $\cos \phi=\hat{n} \cdot \hat{v}$ with $0 \leqslant \phi \leqslant \pi / 2$ and $\alpha$ is an azimuthal angle for $\hat{n}$ in the plane perpendicular to $\vec{v}$ with $0 \leqslant \alpha \leqslant 2 \pi$. We can now simplify the restituting term in the extended LB equation by noting that we can combine the Jacobian with the distribution function $F$ to write

$$
\left|\frac{\partial \boldsymbol{\rho}^{\prime}}{\partial \boldsymbol{\rho}}\right| F\left(\boldsymbol{\rho}^{\prime}\right)=\int d \boldsymbol{\rho}^{\prime} \delta\left(\boldsymbol{\rho}-\boldsymbol{\rho}\left(\boldsymbol{\rho}^{\prime}\right)\right) F\left(\boldsymbol{\rho}^{\prime}\right),
$$

where the $\delta$ function in the integrand selects the right restituting value of the radius of curvature matrix in accordance with Eq. (52). Putting everything together, we can obtain the extended LB equation as

$$
\begin{aligned}
\frac{\partial F}{\partial t}+ & \vec{v} \cdot \frac{\partial F}{\partial \vec{r}}+v \sum_{i=1}^{d-1} \frac{\partial F}{\partial \rho_{i i}} \\
= & n a^{d-1} \int d \hat{n}|\vec{v} \cdot \hat{n}|\left[\Theta(\vec{v} \cdot \hat{n}) \int d \boldsymbol{\rho}^{\prime} \delta\left(\boldsymbol{\rho}-\boldsymbol{\rho}\left(\boldsymbol{\rho}^{\prime}\right)\right)\right. \\
& \left.\times F\left(\vec{r}, \vec{v}^{\prime}, \boldsymbol{\rho}^{\prime}, t\right)-\Theta(-\vec{v} \cdot \hat{n}) F(\vec{r}, \vec{v}, \boldsymbol{\rho}, t)\right] .
\end{aligned}
$$

In the next section we will use Eq. (57) to compute the KS entropy for two- and three-dimensional Lorentz gases in equilibrium. Before turning to this calculation, we make an observation about the restituting radius of curvature matrix. We note that the diagonal elements of the radius of curvature matrix will grow with time between collisions. Thus the average value of the diagonal elements of the radius of curvature matrix before collision will be on the order of the mean free path between collisions $l$. For low density of scatterers, the mean free path $l$ will be much larger than the radius of the individual scatterers $a$ such that $a / l \sim n a^{d} \ll 1$. This observation will allow us to greatly simplify the $\delta$ function in the restituting collision term in Eq. (57) and thereby simplify the calculations to follow.

\section{EQUILIBRIUM SOLUTIONS OF THE EXTENDED LB EQUATION}

In this section we construct the equilibrium solutions of the extended LB equation (57) in two and three dimensions and from these compute $h_{\mathrm{KS}}$. We begin with the twodimensional case. Here the radius of curvature is a simple scalar and Eq. (57) becomes

$$
\begin{aligned}
& \frac{\partial F}{\partial t}+\vec{v} \cdot \frac{\partial F}{\partial \vec{r}}+v \frac{\partial F}{\partial \rho} \\
&=n a v \int_{-\pi / 2}^{\pi / 2} d \phi \cos \phi\left[\int _ { 0 } ^ { \infty } d \rho ^ { \prime } \delta \left(\rho-\frac{a \cos \phi}{a \cos \phi}\right.\right. \\
&\left.2+\frac{\rho^{\prime}}{\rho}\right) \\
&\left.\quad \times F\left(\vec{r}, \vec{v}^{\prime}, \rho^{\prime}, t\right)-F(\vec{r}, \vec{v}, \rho, t)\right] .
\end{aligned}
$$

To find an equilibrium solution, we look for solutions that do not depend upon time, velocity direction, or position and that become the known equilibrium solution for the LB equation when the integration over $\rho$ is carried out. That is, we look for solutions $F$ of the form 


$$
F(\vec{v}, \rho)=\varphi_{0}(v) \psi(\rho),
$$

where $\varphi_{0}(v)=(2 \pi v V)^{-1} \delta\left(v-v_{0}\right)$ is the normalized, equilibrium spatial and velocity distribution function for the moving particle with constant speed, which we here denote as $v_{0}$, and confined to a volume $V$. Then we require that $\psi(\rho)$ be normalized as

$$
\int_{0}^{\infty} d \rho \psi(\rho)=1
$$

It is an easy matter to obtain an equation for $\psi(\rho)$ that reads

$$
\begin{aligned}
v \frac{\partial \psi}{\partial \rho}= & -2 n a v \psi+n a v \int_{-\pi / 2}^{\pi / 2} d \phi \int_{0}^{\infty} d \rho^{\prime} \\
& \times \delta\left(\rho-\frac{a \cos \phi}{2+\frac{a \cos \phi}{\rho^{\prime}}}\right) \psi\left(\rho^{\prime}\right) .
\end{aligned}
$$

An inspection of the $\delta$ function shows that it vanishes unless $\rho<a / 2$. Therefore, for $\rho \geqslant a / 2$, we have the simple result

$$
\psi(\rho)=A e^{-\rho / \ell} \quad \text { for } \rho \geqslant a / 2,
$$

where $\ell=(2 n a v)^{-1}$ is the mean free path length for the moving particle at low density of scatterers and $A$ is a constant to be determined. To treat the distribution function $\psi(\rho)$ for smaller values of $\rho$ we note that we can require that $\psi(\rho) \rightarrow 0$ as $\rho \rightarrow 0$ since the dynamics will increase the value of $\rho$ during free particle motion and will decrease it to some value, still greater than 0 , at the instant of a collision. Further we can require that $\rho$ be continuous at $\rho=a / 2$ since the extended LB equation does not have an explicit $\delta$ function of the form $\delta(\rho-a / 2)$ on the right-hand side. Finally, we note that the dominant contribution to the $\rho^{\prime}$ integral on the righthand side of Eq. (61) comes from $\rho^{\prime} \sim \ell$. Therefore, the $\delta$ function on the right-hand side of Eq. (61) can be approximated by

$$
\delta\left(\rho-\frac{a \cos \phi}{2+\frac{a \cos \phi}{\rho^{\prime}}}\right) \simeq \delta(\rho-(a \cos \phi) / 2) .
$$

(A more detailed examination of this integral keeping the full $\delta$ function shows that the terms neglected here are of order $\tilde{n} \ln \tilde{n}$ compared to the terms retained, where $\tilde{n}=n a^{2}$.) After inserting this expression in Eq. (61) we find that for $\rho<a / 2$,

$$
v \frac{\partial \psi}{\partial \rho}=2 n a v\left[-\psi(\rho)+\frac{2 \sigma}{a\left(1-\sigma^{2}\right)^{1 / 2}} \int_{0}^{\infty} d \rho^{\prime} \psi\left(\rho^{\prime}\right)\right],
$$

where $\sigma=a \rho / 2$. Since $\psi$ is normalized to one, we find that

$$
\psi(\rho)=(1 / \ell)\left\{1-\left[1-\left(\frac{2 \rho}{a}\right)^{2}\right]^{1 / 2}\right\} \text { for } \rho<a / 2
$$

and, using the normalization condition on $\psi$, we find

$$
\psi(\rho)=(1 / \ell) e^{-\rho / \ell} \text { for } \rho \geqslant a / 2 .
$$

[Comparing Eqs. (62) and (65) one sees that $\psi(\rho)$ apparently has a discontinuity at $\rho=a / 2$. This, however, is an artifact of the low-density approximations we have made. Note that the jump in $\psi$ is of relative order $n a^{2}$ indeed.] Combining this expression for $\psi$ with Eqs. (47) and (59) we obtain

$$
\lambda^{(+)}=h_{\mathrm{KS}}=2 \operatorname{nav}(1-\ln 2-\mathcal{C}-\ln \tilde{n}) \text { for } \tilde{n} \ll 1,
$$

in agreement with the result given by Eq. (26).

Now we turn to the three-dimensional case. This is somewhat more complicated than the two-dimensional case since $\boldsymbol{\rho}$ is a $2 \times 2$ matrix and not a scalar. However, we can still simplify the $\delta$ function in the restituting collision integral by noticing that the diagonal elements of the curvature matrix grow with time during the free steaming intervals between collisions. Consequently, the diagonal elements of $\boldsymbol{\rho}^{\prime}$ appearing on the left-hand side of Eq. (54) are of the order of the mean free path length immediately before a collision with a scatterer. An elementary consideration of the properties of the inverses of $2 \times 2$ matrices with large diagonal elements shows that the dominant contribution to the radius of curvature matrix $\boldsymbol{\rho}$ comes from setting the left-hand side of Eq. (54) equal to zero. This greatly simplifies the $\delta$ function appearing in the collision integral on the right-hand side of Eq. (57). The effect of this simplification is that we have neglected terms of relative order $a / \ell$, which are density corrections to the terms we keep.

The equilibrium distribution function $F(\vec{v}, \boldsymbol{\rho})$ can be factorized as

$$
F(\vec{v}, \boldsymbol{\rho})=\varphi(v) \psi(\boldsymbol{\rho})
$$

where $\varphi(v)=\left(4 \pi^{2} v_{0}^{2} V\right)^{-1} \delta\left(v-v_{0}\right)$ is the normalized equilibrium distribution function for the moving particle. The extended LB equation reduces to

$$
\begin{aligned}
& v\left(\frac{\partial}{\partial \rho_{11}}+\frac{\partial}{\partial \rho_{22}}\right) \psi(\boldsymbol{\rho}) \\
& =-n a^{2} \pi v \psi(\boldsymbol{\rho})+n a^{2} v \int_{0}^{\pi / 2} d \phi \int_{0}^{2 \pi} d \alpha \int d \rho_{11}^{\prime} \int d \rho_{12}^{\prime} \\
& \quad \times \int d \rho_{21}^{\prime} \int d \rho_{22}^{\prime} \sin \phi \cos \phi \\
& \quad \times \prod_{i, j} \delta\left(\rho_{i j}-\rho_{i j}(\phi, \alpha)\right) \psi\left(\boldsymbol{\rho}^{\prime}\right) .
\end{aligned}
$$

The matrix elements of $\boldsymbol{\rho}(\phi, \alpha)$ can be obtained by solving Eq. (54) under the approximation $\left[\boldsymbol{\rho}^{\prime}\right]^{-1}=0$, which can be justified again as a low-density approximation by the same arguments as in the two-dimensional case. The results are

$$
\rho_{11}(\phi, \alpha)=\frac{a \cos \phi}{2}\left(1+\tan ^{2} \phi \sin ^{2} \alpha\right),
$$




$$
\begin{gathered}
\rho_{12}(\phi, \alpha)=\rho_{21}(\phi, \alpha)=-\frac{a \sin ^{2} \phi \sin 2 \alpha}{4 \cos \phi} \\
\rho_{22}(\phi, \alpha)=\frac{a \cos \phi}{2}\left(1+\tan ^{2} \phi \cos ^{2} \alpha\right) .
\end{gathered}
$$

The restituting term in Eq. (69) contains an integration of $\psi\left(\boldsymbol{\rho}^{\prime}\right)$ over its arguments and we can set

$$
\int d \boldsymbol{\rho}^{\prime} \psi\left(\boldsymbol{\rho}^{\prime}\right)=1
$$

We use $\psi$ to compute the average value of $\operatorname{Tr}(\boldsymbol{\rho})^{-1}$ $=[\operatorname{Tr} \boldsymbol{\rho}][\operatorname{det} \boldsymbol{\rho}]^{-1}$, which determines the sum of the positive Lyapunov exponents. The $\rho_{i j}(\phi, \alpha)$ occurring in the $\delta$ function on the right-hand side of Eq. (69) can be identified at low density with the values of $\rho_{i j}$ right after a collision with collision parameters $\phi$ and $\alpha$. From Eq. (71) it follows that these values are always equal for $\rho_{12}$ and $\rho_{21}$. Furthermore, these quantities do not change in between collisions, so we may set $\psi(\boldsymbol{\rho})=f\left(\rho_{11}, \rho_{22}, \rho_{12}\right) \delta\left(\rho_{12}-\rho_{21}\right)$. Next we change variables from $\rho_{11}, \rho_{22}, \rho_{12}$ to $\rho_{1}, \rho_{2}, \rho_{12}$, where $\rho_{1}, \rho_{2}$ are the eigenvalues of the $2 \times 2$ matrix $\boldsymbol{\rho}$. The Jacobian for the transformation of variables, given by

$$
J\left(\frac{\rho_{1}, \rho_{2}}{\rho_{11}, \rho_{22}}\right)=\frac{\left|\rho_{1}-\rho_{2}\right|}{\left[\left(\rho_{1}-\rho_{2}\right)^{2}-4 \rho_{12}^{2}\right]^{1 / 2}},
$$

will be included in the integrand in Eq. (78). After some straightforward algebra we obtain the following equation for $g\left(\rho_{1}, \rho_{2}, \rho_{12}\right) \equiv f\left(\rho_{11}, \rho_{22}, \rho_{12}\right)$ :

$$
\begin{aligned}
v\left(\frac{\partial}{\partial \rho_{1}}\right. & \left.+\frac{\partial}{\partial \rho_{2}}\right) g\left(\rho_{1}, \rho_{2}, \rho_{12}\right)+\nu g\left(\rho_{1}, \rho_{2}, \rho_{12}\right) \\
= & \frac{\nu}{2} \int_{0}^{\pi / 2} d \phi \int_{0}^{2 \pi} d \alpha(\sin \phi)(\cos \phi)|\cos 2 \alpha| \\
& \times \delta\left(\rho_{1}-\frac{a}{2 \cos \phi}\right) \delta\left(\rho_{2}-(a \cos \phi) / 2\right) \\
& \times \delta\left(\rho_{12}+\left(a \cos \phi \tan ^{2} \phi \sin 2 \alpha\right) / 4\right),
\end{aligned}
$$

where $\nu=n \pi a^{2} v$ is the average collision frequency for the moving particle. The $\alpha$ integration can be carried out and we see that $g$ can be written in the form

$$
g\left(\rho_{1}, \rho_{2}, \rho_{12}\right)=\Theta\left(1-\frac{2\left|\rho_{12}\right|}{\left|\rho_{1}-\rho_{2}\right|}\right) h\left(\rho_{1}, \rho_{2}\right)
$$

where $h$ satisfies

$$
v\left(\frac{\partial}{\partial \rho_{1}}+\frac{\partial}{\partial \rho_{2}}\right) h\left(\rho_{1}, \rho_{2}\right)+\nu h\left(\rho_{1}, \rho_{2}\right)
$$

$$
\begin{aligned}
& =\frac{4 \nu}{\pi} \int_{0}^{\pi / 2} d \phi \frac{\cos ^{2} \phi}{\sin \phi} \delta\left(\rho_{2}-(a \cos \phi) / 2\right) \\
& \quad \times \delta\left(\rho_{1}-a(2 \cos \phi)^{-1}\right) .
\end{aligned}
$$

We can now express the sum of the positive Lyapunov exponents in terms of $h$ as

$$
\begin{aligned}
\lambda_{\max }^{+}+\lambda_{\min }^{+}= & \int d \rho_{1} \int d \rho_{2} \int d \rho_{12}\left(\frac{1}{\rho_{1}}+\frac{1}{\rho_{2}}\right) \\
& \times\left(\frac{\left|\rho_{1}-\rho_{2}\right|}{\left[\left(\rho_{2}-\rho_{1}\right)^{2}-4 \rho_{12}^{2}\right]^{1 / 2}}\right) \\
& \times \Theta\left(1-\frac{2\left|\rho_{12}\right|}{\left|\rho_{1}-\rho_{2}\right|}\right) h\left(\rho_{1}, \rho_{2}\right) .
\end{aligned}
$$

The $\rho_{12}$ integration can be carried out easily, yielding

$$
\lambda_{\max }^{+}+\lambda_{\min }^{+}=\frac{\pi}{2} \int d \rho_{1} \int d \rho_{2}\left(\frac{1}{\rho_{1}}+\frac{1}{\rho_{2}}\right)\left|\rho_{2}-\rho_{1}\right| h\left(\rho_{1}, \rho_{2}\right) \text {. }
$$

We define a new function $p\left(\rho_{1}, \rho_{2}\right)=(\pi / 2) \mid \rho_{2}$ $-\rho_{1} \mid h\left(\rho_{1}, \rho_{2}\right)$, which satisfies

$$
\begin{aligned}
v\left(\frac{\partial}{\partial \rho_{1}}\right. & \left.+\frac{\partial}{\partial \rho_{2}}\right) p\left(\rho_{1}, \rho_{2}\right)+\nu p\left(\rho_{1}, \rho_{2}\right) \\
= & 2 \nu \int_{0}^{\pi / 2} d \phi \frac{\cos ^{2} \phi}{\sin \phi}\left(\frac{1}{\cos \phi}-\cos \phi\right) \\
& \times \delta\left(\rho_{2}-(a \cos \phi) / 2\right) \delta\left(\rho_{1}-a(2 \cos \phi)^{-1}\right) .
\end{aligned}
$$

Introducing $\quad p_{1}(\rho)=\int_{0}^{\infty} d \rho_{2} p\left(\rho, \rho_{2}\right) \quad$ and $\quad p_{2}(\rho)$ $=\int_{0}^{\infty} d \rho_{1} p\left(\rho_{1}, \rho\right)$ enables us to express the sum of the Lyapunov exponents very simply as

$$
\lambda_{\max }^{+}+\lambda_{\min }^{+}=\sum_{i=1,2} \int_{0}^{\infty} d \rho \frac{1}{\rho} p_{i}(\rho),
$$

where the $p_{i}(\rho)$ satisfy

$$
\begin{aligned}
v \frac{\partial p_{1}(\rho)}{\partial \rho}+\nu p_{1}(\rho)= & 2 \nu \int_{0}^{\pi / 2} d \phi(\sin \phi)(\cos \phi) \\
& \times \delta(\rho-(a \cos \phi) / 2), \\
v \frac{\partial p_{2}(\rho)}{\partial \rho}+\nu p_{2}(\rho)= & 2 \nu \int_{0}^{\pi / 2} d \phi(\sin \phi)(\cos \phi) \\
& \times \delta\left(\rho-a(2 \cos \phi)^{-1}\right) .
\end{aligned}
$$

Solving Eqs. (82) and (83) for $p_{1}, p_{2}$ and inserting the solution into Eq. (81), one obtains an expression for the sum of the positive Lyapunov exponents that agrees with Eq. (28). It is worth mentioning that we can solve Eq. (75) to provide $g\left(\rho_{1}, \rho_{2}, \rho_{12}\right)$ as an explicit function of the variables $\rho_{1}, \rho_{2}, \rho_{12}$ using the method of characteristics. As this solution will prove useful in subsequent papers, we outline the 
method in Appendix B. There we also briefly indicate how the individual Lyapunov exponents can be calculated using $g\left(\rho_{1}, \rho_{2}, \rho_{12}\right)$ and simple results from the theory for eigenvalues of products of random matrices [24]. While the results obtained with the extended LB equation properly agree with those obtained by more direct kinetic theory arguments, we will need to use the extended LB equation in order to obtain the Lyapunov exponents and KS entropies for the spatially inhomogeneous systems that occur when one considers escape-rate methods for connecting chaotic quantities with transport coefficients. This will be treated elsewhere.

\section{THE NEGATIVE LYAPUNOV EXPONENTS AND THE ANTI-LORENTZ BOLTZMANN EQUATION}

We now turn our attention to the Lyapunov exponents that characterize the exponential convergence of trajectories on a stable manifold in the $(2 d-1)$-dimensional constant energy surface in the phase space of the moving particle. We recall that two arbitrary but infinitesimally nearby trajectories will certainly separate eventually with time. We have used this fact to derive formulas and explicit expressions for the positive Lyapunov exponents. However, Liouville's theorem showing that the measure of a small region of phase space is constant in time as one follows the motion of points initially in that region implies that there must be a compensating set of negative Lyapunov exponents that act in concert with the positive ones to keep phase-space measures constant in time. Moreover, the fact that the Lorentz gas is a symplectic Hamiltonian system has as a consequence the existence of a conjugate pairing rule [19]. That is, for such a system, the Lyapunov exponents come in positive and negative pairs such that the sum of each corresponding pair is zero. Thus, in this case at least, the calculation of the negative Lyapunov exponents is trivial: They are just the opposites of the positive ones. However, in future work where we plan to treat thermostated systems, this form of the conjugate pairing rule no longer holds [29-31] and we will need to find methods to compute both positive and negative exponents individually.

The most obvious way to obtain the negative Lyapunov exponents is to compute the positive Lyapunov exponents for the time-reversed motion. Upon time reversal trajectories that approach each other in the forward motion will separate. In fact, almost all trajectories will separate in both the forward and the time reversed direction, but in general (i.e., for nonsymplectic systems) with different exponents. In the forward motion they will separate with rates given by the positive Lyapunov exponents and in the time-reversed motion with rates equal to the magnitudes of the negative Lyapunov exponents. Thus, to calculate the negative exponents we consider the binary collision dynamics already discussed before, but look at the time-reversed motion. If, in the forward-time direction, the moving particle is uncorrelated with a scatterer before collision, in the time-reversed motion it will be uncorrelated with the same scatterer after the collision. We therefore should consider a kind of backward kinetic theory, where the particles are uncorrelated with the scatterers after their collisions instead of before them. This will differ in important respects from the ordinary Lorentz-Boltzmann equation. In order to illustrate this we consider first a calculation of the sum of the negative Lyapunov exponents for the

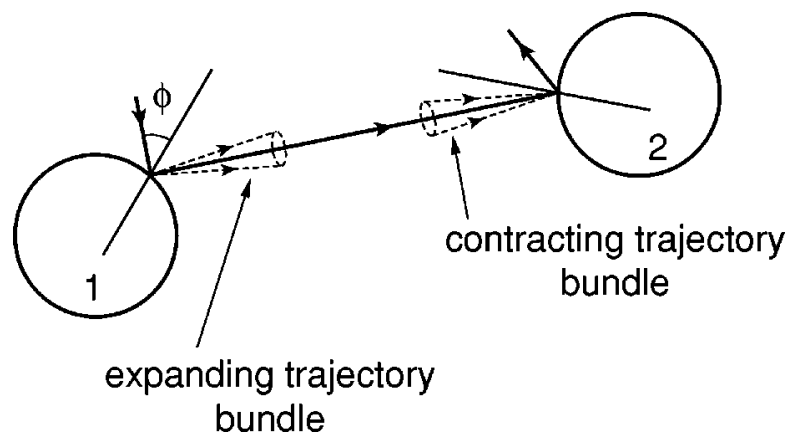

FIG. 1. Trajectory from scatterer 1 to scatterer 2 with bundles of expanding and contracting trajectories indicated.

two-dimensional (2D) and 3D dilute Lorentz gases, using a simple kinetic theory argument similar to that used in Sec. II.

\section{A. A simple kinetic theory method for the sum of the negative Lyapunov exponents}

We first consider the 2D case. We wish to follow an infinitesimal trajectory bundle that is contracting and remains contracting for all future times. Such a bundle is illustrated in Fig. 1. We follow the motion of this bundle from scatterer 1 to scatterer 2 . In order that the bundle remain contracting after the collision with scatterer 2 we require that the radius of curvature of this bundle be very close (with corrections of order $n a^{2}$ ) to the value $a \cos \phi / 2$ just before the collision with scatterer 2 . We denote the direction of the velocity of the moving particle just after the collision with scatterer 1 by the angle $\theta$ with respect to some space fixed axis and compute the negative Lyapunov exponent in the following way. Since we know that the radius of curvature just before the collision with 2 is $a \cos \phi / 2$, the radius of curvature just after the collision with 1 must be $a \cos \phi / 2+v t$, where $t$ is the time interval between the collision with 1 and 2 . From this it follows that

$$
v \int_{0}^{t} d \tau[\rho(\tau)]^{-1}=\ln \left[\frac{2 v t}{a \cos \phi}\right]
$$

where $\rho(\tau)$ is the radius of curvature of the contracting bundle at times $0 \leqslant \tau \leqslant t$ before the collision with 2 and $v t$ $\gg a$. The time average of this expression corresponds to the result obtained by combining Eqs. (17) and (21), in agreement with the conjugate pairing rule for symplectic systems. In the case of a thermostated system however, Eqs. (21) and (84) are replaced by expressions that depend on the velocity angle $\theta$ in different ways. As a consequence, the positive and negative Lyapunov exponents are no longer each other's opposite.

The three-dimensional case proceeds in exactly the same way. We follow an infinitesimal contracting trajectory bundle from a collision with scatterer 1 to a collision with scatterer 2 such that before the collision with scatterer 2 , the radius of curvature matrix is given by $[(2 \cos \phi \mathbf{P}) / a]^{-1}$, where $\mathbf{P}$ is defined in Eq. (55). We then find easily that if $t$ is the time interval between the collisions of the moving particle with scatterers 1 and 2, then the radius of curvature matrix at some time $\tau$ between zero and $t$ after the collision with scatterer 1 is 


$$
\boldsymbol{\rho}(\tau)=v(t-\tau) \mathbf{1}+\frac{a}{2 \cos \phi} \mathbf{P}^{-1}
$$

For calculating the sum of the positive Lyapunov exponents one needs the average again of $\operatorname{Tr}[\boldsymbol{\rho}(\tau)]^{-1}$, but now under the Stosszahlansatz for the postcollisional (under the time reversed dynamics, so in reality the precollisional) coordinates. However, due to the time-reversal symmetry of the dynamics the distribution of the postcollisional coordinates $\phi^{\prime}$ and $\alpha^{\prime}$ is the same as that of $\phi$ and $\alpha$ and of course the distribution of intercollisional times also is the same for forward and backward motion. Therefore, the averaging procedure yields the same results as for Eq. (38) and the sum of the negative Lyapunov exponents is given by the opposite of Eq. (28).

The individual Lyapunov exponents can be obtained using results from the theory for eigenvalues of products of random matrices, as described in Appendix B. The results are, as expected, that each negative exponent is paired with a positive one such that their sum is zero. Now we turn to the method of distribution functions for calculating the negative Lyapunov exponents.

\section{B. The extended anti-Lorentz-Boltzmann equation}

In order to use distribution functions to compute the negative Lyapunov exponents or their sum, we need to construct a Boltzmann-like equation for the time-reversed motion. If one reviews the derivation of the Boltzmann equation, one sees that the colliding particles are taken to be uncorrelated before the direct or the restituting collisions. If we were to look at the time-reversed motion, the terms "before" and "after" are interchanged and for the time-reversed motion the colliding particles are uncorrelated after the collisions rather than before. That is, referring to Fig. 1 again, the timereversed motion has a collision of the moving particle with scatterer 2 , followed by a collision of the particle with scatterer 1 . Before the collision with 2 , the moving particle is correlated with 2 , but not so after the collision. After the collision, the radius of curvature or the radius of curvature matrix is given by the values $a \cos \phi / 2$ in two dimensions, or $[2 \cos \phi \mathbf{P} / a]^{-1}$ in three dimensions. The radius of curvature (in two dimensions) or the diagonal elements of the matrix (in three dimensions) grow over the time interval $t$ until the collision with 1 . Since the moving particle is correlated with the scatterers before the collisions and not after them, when deriving a Boltzmann-like equation for the distribution function, we must apply the Stosszahlansatz to the exiting collision cylinders after the collisions and not to the entering cylinders before collision, as is the usual case.

In order to clarify this procedure we first consider a derivation of the anti-Lorentz-Boltzmann equation (ALBE) for the distribution function $f_{-}(\vec{r}, \vec{v}, t)$ for the position and velocity of the moving particle at time $t$. We will then generalize this derivation by adding the radius of curvature variables. Since there is no particular problem with the time reversal of the free streaming of the particle, we can write the ALBE in the form

$$
\frac{\partial f_{-}(\vec{r}, \vec{v}, t)}{\partial t}+\vec{v} \cdot \frac{\partial f_{-}}{\partial \vec{r}}=\Gamma_{-}^{+}-\Gamma_{-}^{-},
$$

where $\Gamma_{-}^{+}$represents the rate at which particles are produced at $\vec{r}$ with velocity $\vec{v}$ and $\Gamma_{-}^{-}$represents the rate at which such particles are lost. Noting the fact that we should apply the Stosszahlansatz to the exiting collision cylinders, we see that the rate at which particles with velocity $\vec{v}$ are produced is

$$
\Gamma_{-}^{+} \delta \vec{r} \delta \vec{v} \delta t=n a^{d-1} \int d \hat{n}(\hat{n} \cdot \vec{v}) f_{-}(\vec{r}, \vec{v}, t) \delta \vec{r} \delta \vec{v} \delta t,
$$

where $\hat{n} \cdot \vec{v} \geqslant 0$. That is, particles with velocity $\vec{v}^{\prime}=\vec{v}$ $-2(\hat{n} \cdot \vec{v}) \hat{n}$ collide with scatterers and produce particles with velocity $\vec{v}$, but the distribution function we need to calculate the rate of these collisions for is not $f_{-}\left(\vec{r}, \vec{v}^{\prime}, t\right)$, but $f_{-}(\vec{r}, \vec{v}, t)$. Similarly,

$$
\Gamma_{-}^{-}=n a^{d-1} \int d \hat{n}\left(\hat{n} \cdot \vec{v}^{\prime}\right) f_{-}\left(\vec{r}, \vec{v}^{\prime}, t\right),
$$

where $\hat{n} \cdot \vec{v}^{\prime} \geqslant 0$ since particles with velocity $\vec{v}^{\prime}$ are produced when a particle of velocity $\vec{v}$ collides with a scatterer with collision vector $\hat{n}$. Putting these terms together, we obtain

$$
\begin{aligned}
\frac{\partial f_{-}(\vec{r}, \vec{v}, t)}{\partial t}+\vec{v} \cdot \frac{\partial f_{-}}{\partial \vec{r}}= & n a^{d-1} \int d \hat{n}|\vec{v} \cdot \hat{n}|\left[f_{-}(\vec{r}, \vec{v}, t)\right. \\
& \left.-f_{-}\left(\vec{r}, \vec{v}^{\prime}, t\right)\right],
\end{aligned}
$$

where we have used the fact that $|\vec{v} \cdot \hat{n}|=\left|\vec{v}^{\prime} \cdot \hat{n}\right|$ and the integration in Eq. (89) is over a semicircle (for $d=2$ ) or a hemisphere (for $d=3$ ). This equation looks exactly like the Lorentz-Boltzmann equation (LBE) (45), except for the fact that the collision integral has the opposite signs in Eqs. (89) and (45) [32]. The ALBE (89) has the usual equilibrium distribution function as a stationary solution; although it is a highly unstable solution, any deviation will tend to grow exponentially in time. In fact, for this reason it is an ill-posed equation, as arbitrarily small initial deviations can grow at arbitrarily large rates and therefore its solution is not well defined. This ambiguity can be removed by requiring that the actual solution describing a physical system after a long enough time that all these rapidly decaying solutions (observed in the forward time direction) have died out is the time reverse of the solution of the ordinary LBE (in a closed isolated system or system coupled to a single heat bath this will become the equilibrium solution for long times). So there is little use in employing the ALBE as such since to obtain its physically relevant solution one has to solve the ordinary LBE anyway. The extension of the ALBE discussed in the following paragraphs, on the other hand, does provide a useful tool for calculating negative Lyapunov exponents.

To this end we need to include again the radius of curvature matrix as variables in the distribution function $F_{-}(\vec{r}, \vec{v}, \boldsymbol{\rho}, t)$. The equation for $F_{-}$is constructed as before, i.e., we write

$$
\frac{\partial F_{-}}{\partial t}+\vec{v} \cdot \frac{\partial F_{-}}{\partial \vec{r}}+v \sum_{i=1}^{d-1} \frac{\partial F_{-}}{\partial \rho_{i i}}=\Gamma_{-}^{+}(\boldsymbol{\rho})-\Gamma_{-}^{-}(\boldsymbol{\rho}) .
$$


To compute $\Gamma_{-}^{+}(\boldsymbol{\rho})$ we multiply the rate at which particles with velocity $\vec{v}$ are produced no matter what their radius of curvature matrix might be, with the fraction of particles with velocity $\vec{v}^{\prime}$ that produce particles with radius of curvature $\boldsymbol{\rho}$ after collision. That is,

$$
\begin{aligned}
\Gamma_{-}^{+}(\boldsymbol{\rho})= & n a^{d-1} \int d \hat{n}|\vec{v} \cdot \hat{n}| \int d \boldsymbol{\rho}^{\prime} F_{-}\left(\vec{r}, \vec{v}, \boldsymbol{\rho}^{\prime}, t\right) \\
& \times\left[\int d \boldsymbol{\rho}^{\prime \prime} F_{-}\left(\vec{r}, \vec{v}^{\prime}, \boldsymbol{\rho}^{\prime \prime}, t\right) \delta\left(\boldsymbol{\rho}-\boldsymbol{\rho}\left(\boldsymbol{\rho}^{\prime \prime}\right)\right)\right] \\
& \times\left[\int d \boldsymbol{\rho}^{\prime \prime} F_{-}\left(\vec{r}, \vec{v}^{\prime}, \boldsymbol{\rho}^{\prime \prime}, t\right)\right]^{-1} .
\end{aligned}
$$

Similarly, to compute $\Gamma_{-}^{-}(\boldsymbol{\rho})$ we multiply the rate at which particles with velocity $\vec{v}^{\prime}$ are produced due to collisions of particles of velocity $\vec{v}$ with scatterers by the fraction of particles of velocity $\vec{v}$ that have the radius of curvature matrix $\boldsymbol{\rho}$. That is,

$$
\begin{aligned}
\Gamma_{-}^{-}(\boldsymbol{\rho})= & n a^{d-1} \int d \hat{n}|\hat{n} \cdot \vec{v}| \int d \boldsymbol{\rho}^{\prime} F_{-}\left(\vec{r}, \vec{v}^{\prime}, \boldsymbol{\rho}^{\prime}, t\right) F(\vec{r}, \vec{v}, \boldsymbol{\rho}, t) \\
& \times\left[\int d \boldsymbol{\rho}^{\prime} F_{-}\left(\vec{r}, \vec{v}, \boldsymbol{\rho}^{\prime}, t\right)\right]^{-1} .
\end{aligned}
$$

We can now assemble all of these results into an extended anti-Lorentz-Boltzmann equation (EALBE), which reads

$$
\begin{aligned}
\frac{\partial F_{-}}{\partial t}+\vec{v} \cdot \frac{\partial F_{-}}{\partial \vec{r}}+v \sum_{i=1}^{d-1} \frac{\partial F_{-}}{\partial \rho_{i i}}= & n a^{d-1} \int d \hat{n}|\vec{v} \cdot \hat{n}| \int d \boldsymbol{\rho}^{\prime} F_{-}\left(\vec{r}, \vec{v}, \boldsymbol{\rho}^{\prime}, t\right)\left[\int d \boldsymbol{\rho}^{\prime \prime} F_{-}\left(\vec{r}, \vec{v}^{\prime}, \boldsymbol{\rho}^{\prime \prime}, t\right) \delta\left(\boldsymbol{\rho}-\boldsymbol{\rho}\left(\boldsymbol{\rho}^{\prime \prime}\right)\right)\right] \\
& \times\left[\int d \boldsymbol{\rho}^{\prime \prime} F_{-}\left(\vec{r}, \vec{v}^{\prime}, \boldsymbol{\rho}^{\prime \prime}, t\right)\right]^{-1}-n a^{d-1} \int d \hat{n}|\hat{n} \cdot \vec{v}| \int d \boldsymbol{\rho}^{\prime} F_{-}\left(\vec{r}, \vec{v}^{\prime}, \boldsymbol{\rho}^{\prime}, t\right) F(\vec{r}, \vec{v}, \boldsymbol{\rho}, t) \\
& \times\left[\int d \boldsymbol{\rho}^{\prime} F_{-}\left(\vec{r}, \vec{v}, \boldsymbol{\rho}^{\prime}, t\right)\right]^{-1}
\end{aligned}
$$

Here too $\hat{n}$ is integrated over a semicircle or a hemisphere. This complicated and nonlinear looking equation [in fact, one should first solve the ALBE, substitute its solution for $\int d \boldsymbol{\rho}^{\prime} F_{-}\left(\vec{r}, \vec{v}^{\prime}, \boldsymbol{\rho}^{\prime}, t\right)$ in Eq. (93), and then solve the EALBE; both equations to be solved then are linear] will form the basis of the calculation of negative Lyapunov exponents and their sums in the more complicated cases to be studied in the future. We should note here that without further conditions this equation, like the ALBE discussed above, constitutes an ill-posed problem. The additional condition that regularizes its solution is that the integral over $\boldsymbol{\rho}$ of the distribution function yields the time reverse of the solution of the ordinary LBE. In the equilibrium case considered here the equation simplifies enormously. In fact, if we use the condition that the system is in a spatially homogeneous equilibrium state, that the integral of the distribution function $F_{-}$over all elements of the radius of curvature matrix is the equilibrium distribution function $\varphi$, which is independent of the velocity direction, we immediately obtain Eq. (69) for the spatially homogeneous case. Therefore, for this equilibrium situation, $F$ - produces the same Lyapunov exponents as in the forward motion, with the exception of the appropriate change of sign due to the time-reversed nature of the motion considered here.

\section{COMPARISON WITH SIMULATIONS AND DISCUSSION}

We have found that the quantities calculated in this paper, the Lyapunov exponents and the KS entropies, expressed as functions of the dimensionless density $\widetilde{n}$, have the general form

$$
\lambda_{i}=A \nu[-\ln \tilde{n}+B+o(1)],
$$

$$
h_{\mathrm{KS}}=A \nu[-\ln \tilde{n}+B+o(1)],
$$

where $\nu$ and $\widetilde{n}$ are the collision frequency and reduced density, given by $\nu=2 n a v$ and $\widetilde{n}=n a^{2}$ in two dimensions and $\nu=\pi n a^{2} v$ and $\widetilde{n}=\pi n a^{3}$ in three dimensions, and $A, B$ are constants that we have determined. In Table I we compare the theoretical results for $A$ and $B$ with values for the same coefficients, as obtained by Dellago and Posch [17] from computer simulations of two- and three-dimensional hardsphere Lorentz gases. The results are in excellent agreement for the coefficient $A$ and there are minor discrepancies in the $B$ values, probably due to the fact that the simulation analysis is difficult at the low densities where the theoretical analysis given here applies.

It is remarkable that the Lyapunov exponents for the three-dimensional completely isotropic random Lorentz gas are different at all. In fact, on the basis of the results obtained in leading order in the density, it has been conjectured [8] that all positive as well as all negative Lyapunov exponents are equal. The methods used in our approach allow a very transparent explanation for the differences, now also confirmed numerically, between the Lyapunov exponents. In particular it becomes clear why all Lyapunov exponents coincide in leading order but differ in next to leading order. The reason for this is related to the different nature of the terms contributing to the different orders [14]. The terms proportional to $n \ln (n / 2)+\mathcal{C}$ in Eqs. (27) and (29) result from averaging over functions, which only depend on the time of free flight. Due to the isotropy of the free flight, this has to lead to equal Lyapunov exponents. The differences arise on averaging over functions, which depend on the collision parameters $\phi$ and $\alpha$. To understand how the scattering process, which is isotropic for a single trajectory, can cause the 
Lyapunov exponents to be different, it is worthwhile considering scattering events in more detail.

For calculating the Lyapunov exponents we have to analyze the scattering of two close by trajectories. Therefore, it becomes possible for a given scattering angle $\phi$ to distinguish whether $\delta \vec{r}_{\perp}$ is in the plane $\mathcal{P}$ spanned by the normal $\hat{n}$ on the sphere and the impact velocity $\vec{v}$ or perpendicular to $\mathcal{P}$. In this way the isotropy of the scattering process becomes effectively broken. This is reflected in the eigenvalue structure of the radius of curvature matrix $\boldsymbol{\rho}^{+}=(2 \cos \phi \mathbf{P} / a)^{-1}$ [see Eq. (55)]. There are two eigenvalues $\rho_{1}=a \cos \phi / 2$ and $\rho_{2}=a /(2 \cos \phi)$. Here the eigenvalue $\rho_{1}$ corresponds to the eigendirection $\vec{e}_{1}$, which is in the plane $\mathcal{P}$, and the eigenvalue $\rho_{2}$ corresponds to the eigendirection that is perpendicular to the plane $\mathcal{P}$, with both eigendirections perpendicular to $\vec{v}$. Note that for $\phi=0$ the eigenvalues are the same. This can be understood by realizing that for $\phi=0$ the particle hits the sphere head on, i.e., $\vec{v}$ is parallel to $\hat{n}$. For this special case the two eigendirections are clearly equivalent, but for other values of $\phi$ this symmetry is lost and the eigenvalues differ. Therefore, we may conclude that the lack of degeneracy of the positive Lyapunov exponents is due to the lack of rotational symmetry when the nearby trajectories hit the sphere.

We conclude with a number of remarks. (i) The results given here can be extended to higher densities in a number of ways. In particular, BBGKY hierarchy methods are being developed to provide a systematic density expansion of the Lyapunov exponents and the KS entropies beyond the lowdensity results obtained here. This will be especially important when nonequilibrium situations are considered since there one may see the effects of long-time tail phenomena on the chaotic properties of the system.

(ii) As remarked earlier, we relied upon the low dimensionality of our systems to obtain all of the relevant Lyapunov exponents. For a four-dimensional Lorentz gas we would need to use more sophisticated techniques to obtain all of the Lyapunov exponents since the methods given here could only provide values for the largest exponent and the sum of all of three of the positive exponents. We could not then resolve the two smaller positive exponents, but only could get their sum.

(iii) We have not analyzed here a particularly interesting quantity that gives a more general characterization of the chaotic properties of the system, namely, the Ruelle, or topological, pressure [33,34]. This quantity has the formal structure of an equilibrium free energy and it depends upon a temperaturelike parameter $\beta$. The results obtained here characterize the chaotic properties in the neighborhood of $\beta=1$. Elsewhere it has been shown that for the Lorentz gas, where the disorder is static, in the thermodynamic limit the topological pressure exhibits a localization transition as a function of $\beta$; it is dominated by contributions from particles that are localized within the largest dense cluster for $\beta<1$ and in the largest region without scatterers for $\beta>1$ [35]. It would be very valuable to obtain these results using kinetic theory methods in addition to the more rigorous analysis given in Ref. [35].

(iv) It should be straightforward to extend the results obtained here to other potentials of interaction between the moving particle and the fixed scatterers, at least for dilute
TABLE I. Comparison of theoretical and simulation results.

\begin{tabular}{ccc}
\hline \hline Quantity & Theory & Simulation \\
\hline \multicolumn{3}{c}{$2 \mathrm{D}: \lambda^{+}$} \\
\hline$A$ & 1 & $0.995 \pm 0.009$ \\
$B$ & 0.423 & $0.463 \pm 0.083$ \\
\hline \multicolumn{3}{c}{$3 \mathrm{D}: \lambda_{\max }^{+}$} \\
\hline$A$ & 1 & $0.990 \pm 0.089$ \\
$B$ & 0.309 & $0.387 \pm 0.746$ \\
\hline \multicolumn{3}{c}{$3 \mathrm{D}: \lambda_{\min }^{+}$} \\
\hline$B$ & 1 & $0.992 \pm 0.084$ \\
\hline & -0.077 & $-0.015 \pm 0.715$ \\
\hline$A$ & $3 \mathrm{D}: h_{\mathrm{KS}}$ \\
\hline
\end{tabular}

systems. It is well known that the Boltzmann transport equation can be applied to gases that interact with other than hard-core potentials [18]. In fact, a wide variety of interaction potentials may be used in the Boltzmann equation to determine the transport properties of the corresponding gases. Certainly Lyapunov exponents can be calculated for Lorentz gases where the moving particle has other than hardcore interactions with the scatterers as well.

(v) Finally, we mention that the method given here can be adapted to cases where all the particles in the system are moving, namely, a dilute gas. Expressions for the KS entropy [36] and the largest Lyapunov exponent of two- and three-dimensional gases with short-range forces [37] have already been obtained this way.

In the future we plan to extend the results here to open systems with escape and compute the escape rates, Lyapunov exponents, and KS entropies characterizing the fractal repeller that underlies diffusion in open systems with absorbing boundaries. This work will make heavy use of the extended LB equation and we regard the present paper as an introduction future work.

\section{ACKNOWLEDGMENTS}

The authors would like to thank E. G. D. Cohen, Ch. Dellago, M. H. Ernst, H. A. Posch, C. Appert, and R. van Zon for many helpful conversations as this work progressed. Ch. Dellago and H. A. Posch kindly supplied the results of their simulations, which were used in Table I. We thank them as well as C. Ferguson, who carried out the analysis of their data. J.R.D wishes to thank D. Panja for useful conversations as well as the National Science Foundation for support under Grants Nos. PHY-93-21312 and PHY-96-00428. A.L. thanks the DFG for financial support, through Grant No. SFB 262, during the time this paper was written. H.v.B was supported by FOM, SMC, and the NWO Priority Program Non-Linear Systems, which are financially supported by the Nederlandse Organisatie voor Wetenschappelijk Onderzoek. He thanks the ENS, Lyon for its hospitality during part of this project. 


\section{APPENDIX A: BINARY COLLISION DYNAMICS AND THE RADIUS OF CURVATURE MATRIX}

Here we give a brief review of the derivation of the formulas for the change in the spatial and velocity deviations at a binary collision used in Eqs. (6) and (7) based on the method of Dellago, Posch, and Hoover [21]. We relate these formulas to the expression used for the change in the radius of curvature matrix, given by Eq. (35), which in turn was discussed by Gaspard and Dorfman [20]. Consider a trajectory of the particle moving among the scatterers. We denote the initial position and velocity of this trajectory by $x(0)$ and the position and velocity at time $t$ later by $x(t)$. Consider also a trajectory that is obtained by an infinitesimal displacement of the initial position and velocity to $x(0)+\delta x(0)$ and denote the position and velocity of the second trajectory at time $t$ by $x(t)+\delta x(t)$. We require the two trajectories to be infinitesimally close. Our goal is deriving equations for $\delta x(t)=(\delta \vec{r}(t), \delta \vec{v}(t))$. We take all trajectories with the same energy, which leads to the condition that $\delta \vec{v}(t) \cdot \vec{v}(t)=0$, so the velocity deviation is always perpendicular to the velocity $\vec{v}(t)$. We can also set the position deviation $\delta \vec{r}(t) \cdot \vec{v}(t)=0$ since this simply requires that the position deviation is perpendicular to the velocity at the initial time.

Now in between collisions $\delta \vec{r}(t), \delta \vec{v}(t)$ satisfy Eqs. (4) and (5). However, the change in these quantities at collision is more complicated. To analyze this change we suppose that the trajectory with $x(t)$ has a collision with some scatterer at time $\tau$. Then immediately after the collision the velocity has changed to $\vec{v}^{+}=\vec{v}-2(\vec{v} \cdot \hat{n}) \hat{n}$, where $\hat{n}$ is a unit vector in the direction from the center of the scatterer to the point of contact and $\vec{v}$ is the velocity immediately before collision. The displaced trajectory will have a collision at a slightly displaced time $\tau+\delta \tau$ and at a slightly different point on the same scatterer located by unit vector $\hat{n}+\delta \hat{n}$, with $\hat{n} \cdot \delta \hat{n}=0$. By examining the scattering equations for the displaced trajectory one easily finds

$$
\begin{gathered}
\delta \vec{v}^{+}=(\mathbf{1}-2 \hat{n} \hat{n}) \cdot \delta \vec{v}-2[(\vec{v} \cdot \hat{n}) \delta \hat{n}+(\vec{v} \cdot \delta \hat{n}) \hat{n}], \\
\delta \vec{r}(\tau)=-\delta \tau \vec{v}+a \delta \hat{n}, \\
\delta \vec{r}^{+}=-\delta \tau \vec{v}^{+}+a \delta \hat{n} .
\end{gathered}
$$

We use the condition $\hat{n} \cdot \delta \hat{n}=0$ to obtain $\delta \tau$ $=-[\hat{n} \cdot \delta \vec{r}(\tau)] / \vec{v} \cdot \hat{n}$. Simple algebra leads to Eqs. (6) and (7) in the text. It is important to note that $\delta \vec{r}(\tau)$ is the spatial deviation when the "main" trajectory has a collision, while

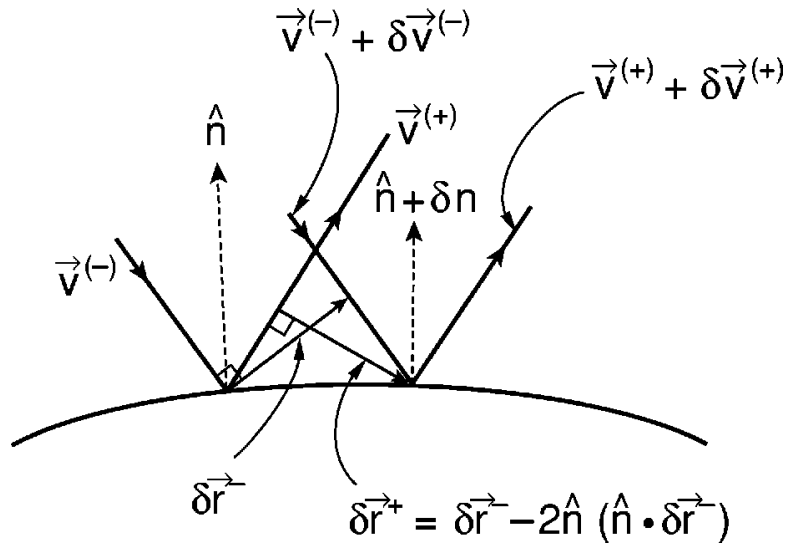

FIG. 2. Arrangement of the position and velocity vectors for one trajectory at the instant of a collision of the moving particle with the scatterer and at the instant of the collision of the particle with the same scatterer along an infinitesimally displaced trajectory.

$\delta \vec{r}^{+}$is the spatial separation at the instant that the displaced trajectory has a collision. (It is easy to visualize this in the case that $\delta \tau>0$, as illustrated in Fig. 2.)

Now we introduce the radius of curvature matrix as given by Eq. (33). We note that $\boldsymbol{\rho}$ is a matrix of rank $d-1$. If we substitute the definition of this matrix [Eq. (33)] into Eq. (6), we obtain

$$
\boldsymbol{\rho}^{+} \cdot \delta \vec{v}^{+}=\mathbf{U} \cdot \boldsymbol{\rho}^{-} \cdot \delta \vec{v}^{-}
$$

where $\mathbf{U}=\mathbf{U}^{-1}=\mathbf{1}-2 \hat{n} \hat{n}$ is a reflection matrix and is discussed in Ref. [20]. Here the superscripts,+- denote after and before collision, respectively. The operators $\mathbf{U}$ have determinant -1 and they ensure the proper orientations, at collision, of the planes in which the radius of curvature matrices are defined. We now substitute Eq. (7) for $\delta \vec{v}^{+}$into Eq. (A4) and use relation (33) and some elementary matrix manipulations to obtain Eq. (35). As mentioned in the main text, the inverse matrices $\left[\boldsymbol{\rho}_{u}^{-1(+)}\right]$ and $\left[\boldsymbol{\rho}_{u}^{-1(-)}\right]$ are defined in the subspaces orthogonal to $\vec{v}$ and $\vec{v}^{\prime}$, respectively.

We can obtain a considerable simplification of the analysis of the spatial deviation vector, $\delta \vec{r}_{\perp}^{(-)}$in Eq. (36) by using the properties of the matrices $\mathbf{U}$. That is, we can easily show that it is possible to express the spatial deviation vector in terms of radius of curvature matrices $\overline{\boldsymbol{\rho}}$, all of which are defined in a plane perpendicular to the initial velocity $\vec{v}(0)$. To see this, consider the right-hand side of Eq. (36) for the case that $n=2$. We have

$$
\delta \vec{r}_{\perp}^{(-)}\left(t_{2}\right)=\left[\mathbf{1}_{\perp}(1)+v \tau_{2,1} \boldsymbol{\rho}_{u}^{-1(+)}\left(t_{1}\right)\right] \cdot \mathbf{U}(1) \cdot\left[\mathbf{1}_{\perp}(0)+v \tau_{1,0} \boldsymbol{\rho}_{u}^{-1}(0)\right] \cdot \delta \vec{r}_{\perp}(0) .
$$

Here $\mathbf{1}_{\perp}(i)$ is a unit operator in the plane perpendicular to the velocity vector after the $i$ th collision. Note that Eq. (35) allows us to write

$$
\overline{\boldsymbol{\rho}}_{u}^{-1(+)}\left(t_{1}\right)=\mathbf{U}(1) \cdot \overline{\boldsymbol{\rho}}_{u}^{-1(+)}\left(t_{1}\right) \cdot \mathbf{U}(1),
$$

where

$$
\begin{aligned}
\overline{\boldsymbol{\rho}}_{u}^{-1(+)}\left(t_{1}\right)= & \boldsymbol{\rho}_{u}^{-1}(0)+\frac{2}{a}\left[\hat{v}(0) \hat{n}_{1}+\hat{n}_{1} \hat{v}(0)-\frac{1}{\hat{v}(0) \cdot \hat{n}_{1}} \hat{n}_{1} \hat{n}_{1}\right. \\
& \left.-\left[\hat{v}(0) \cdot \hat{n}_{1}\right] \mathbf{1}\right] .
\end{aligned}
$$


It is important to note that the operator defined by the terms in large square brackets appearing on the right-hand side of Eq. (A7) is orthogonal from the right and left to $\vec{v}(0)$, as is $\boldsymbol{\rho}_{u}$. Therefore, the operator $\overline{\boldsymbol{\rho}}_{u}^{(+)}$is to be evaluated in the plane perpendicular to $\vec{v}(0)$. Furthermore the unit operator $\mathbf{1}_{\perp}$ (1) appearing in the same set of large square brackets with $\boldsymbol{\rho}_{u}^{-1(+)}\left(t_{1}\right)$ can be written as $\mathbf{U}(1) \cdot \mathbf{1}_{\perp}(0) \cdot \mathbf{U}(1)$. From this it follows that

$$
\begin{aligned}
\delta \vec{r}_{\perp}^{(-)}\left(t_{2}\right)= & \mathbf{U}(1) \cdot\left[\mathbf{1}_{\perp}(0)+v \tau_{2,1}\right. \\
& \left.\times \overline{\boldsymbol{\rho}}_{u}^{-1(+)}\left(t_{1}\right)\right] \cdot\left[\mathbf{1}_{\perp}(0)+\boldsymbol{\rho}_{u}^{-1}(0)\right] \cdot \delta \vec{r}_{\perp}(0)
\end{aligned}
$$

If this procedure is followed through each successive collision, Eq. (36) can easily be written as

$$
\delta \vec{r}_{\perp}^{(-)}\left(t_{n}\right)=\mathbf{U}(n-1) \cdot \mathbf{U}(n-2) \cdots \mathbf{U}(1) \cdot \overline{\delta \vec{r}}_{\perp}^{--)}\left(t_{n}\right)
$$

where

$$
\begin{aligned}
\overrightarrow{\delta \vec{r}}_{\perp}^{(-)}\left(t_{n}\right)= & {\left[\mathbf{1}_{\perp}(0)+\overline{\boldsymbol{\rho}}_{u}^{-1(+)}\left(t_{n-1}\right)\right] \cdots } \\
& \times\left[\mathbf{1}_{\perp}(0)+\boldsymbol{\rho}_{u}^{-1}(0)\right] \cdot \delta \vec{r}_{\perp}(0) .
\end{aligned}
$$

The product of the $\mathbf{U}$ matrices appearing on the right-hand side of Eq. (A9) have determinant \pm 1 , of course, and have no bearing on the exponential growth of the spatial deviation vector. As a result one can carry out all calculations of the Lyapunov exponents and the KS entropy in a coordinate system defined in the plane perpendicular to the initial velocity $\vec{v}(0)$. As a result, all of the $\mathbf{U}$ operators can be dispensed with in the calculation of the Lyapunov exponents provided one uses $\overline{\boldsymbol{\rho}}_{u}$ operators as well as unit operators $\mathbf{1}_{\perp}(0)$. This procedure "unwinds" the trajectory of the moving particle and allows all collisions with the scatterers to be treated in one coordinate system. This is useful when one wants to avoid neglecting the left-hand side of Eq. (54), to treat systems at higher densities, and/or to use the methods of random matrix theory in a convenient way, as we do in the following appendix.

\section{APPENDIX B: THE SOLUTION OF THE EXTENDED LB EQUATION USING THE METHOD OF CHARACTERISTICS AND RANDOM MATRIX METHODS}

Here we indicate how Eq. (69) can be solved as a differential equation in three variables using the method of characteristics [38]. We begin with Eq. (75) and change variables to $\sigma_{1}=2 \rho_{1} / a, \sigma_{2}=2 \rho_{2} / a$, and $\sigma_{12}=2 \rho_{12} / a$. We also set $g\left(\rho_{1}, \rho_{2}, \rho_{12}\right)=8 / a^{3} G\left(\sigma_{1}, \sigma_{2}, \sigma_{12}\right)$ and introduce the scaled density $\widetilde{n}=n \pi a^{3}$. Then the equation for $G$ is

$$
\begin{aligned}
\left(\frac{\partial}{\partial \sigma_{1}}+\frac{\partial}{\partial \sigma_{2}}\right) G+\frac{\tilde{n}}{2} G= & 4 K \Theta\left(1-\sigma_{1}\right) \Theta\left(1-\frac{2\left|\sigma_{12}\right|}{\left|\sigma_{2}-\sigma_{1}\right|}\right) \\
& \times\left(\frac{\sigma_{1}^{2}}{\left(1-\sigma_{1}^{2}\right)}\right) \delta\left(\sigma_{2}-\frac{1}{\sigma_{1}}\right),
\end{aligned}
$$

where $K=\tilde{n} / 2 \pi$. To use the method of characteristics we write

$$
\left(\frac{\partial}{\partial \sigma_{1}}+\frac{\partial}{\partial \sigma_{2}}\right) G=\frac{d}{d s} G,
$$

where

$$
\begin{array}{ll}
\frac{d}{d s} \sigma_{1}=1, & \sigma_{1}=\sigma_{1}^{0}+s \\
\frac{d}{d s} \sigma_{2}=1, & \sigma_{2}=\sigma_{2}^{0}+s \\
\frac{d}{d s} \sigma_{12}=0, & \sigma_{12}=\sigma_{12}^{0} .
\end{array}
$$

This substitution converts the partial differential equation into a simple differential equation that can be solved by elementary means as a function of $s$, once we have specified the appropriate boundary conditions. Further, in the $\left(\sigma_{1}, \sigma_{2}\right)$ plane, the $s$ integration corresponds to an integration along a set of lines given by Eqs. (B3), called the characteristic lines. The inhomogeneous term on the right-hand side of Eq. (B1) is zero everywhere except on the line $\sigma_{2}=1 / \sigma_{1}, \sigma_{1} \leqslant 1$. We look for solutions that vanish at $\sigma_{1}=0$ and at $\sigma_{2}=0$ and we note that the solution $\hat{G}$ of the homogeneous equation, expressed in terms of $s$, has the form

$$
\hat{G}=G_{0} e^{-\widetilde{n s} / 2} .
$$

All of the conditions can be satisfied if $G$ vanishes in the $\left(\sigma_{1}, \sigma_{2}\right)$ plane, except in the region defined by the curves $\sigma_{2} \geqslant \sigma_{1}, \sigma_{1}>1$ and $\sigma_{2} \geqslant 1 / \sigma_{1}, \sigma_{1} \leqslant 1$. See Fig. 3. Choosing $\sigma_{1}^{0}=z \leqslant 1, \sigma_{2}^{0}=1 / \sigma_{1}^{0}$, and $\sigma_{12}=\sigma_{12}^{0}:=\sin (2 \hat{\alpha})\left|\sigma_{2}-\sigma_{1}\right| / 2$, the characteristic lines are given by

$$
\begin{gathered}
\sigma_{1}=z+s, \quad z \leqslant 1 \\
\sigma_{2}=\frac{1}{z}+s, \\
\sigma_{12}=\frac{1-z^{2}}{2 z} \sin (2 \hat{\alpha}) .
\end{gathered}
$$

We then arrive at the equation

$$
\begin{aligned}
\frac{d}{d s} G+\frac{\tilde{n}}{2} G= & K \Theta(1-z) \Theta(2 \pi-\hat{\alpha}) \Theta(\hat{\alpha})\left(\frac{z^{2}}{1-z^{2}}\right) \\
& \times\left(\frac{z^{2}}{1+z^{2}}\right) \delta(s) .
\end{aligned}
$$

The probability density for the random variables $s, z, \hat{\alpha}$ is given by $\hat{f}(s, z, \hat{\alpha})=J(s, z, \hat{\alpha}) G(s, z, \hat{\alpha})$, where $J$ is the Jacobian $J=\left|\partial\left(\sigma_{1}, \sigma_{2}, \sigma_{12}\right) / \partial(s, z, \hat{\alpha})\right|=\left(1-z^{2}\right)\left(1+z^{2}\right) / z^{3} . \hat{f}$ obeys the equation

$$
\frac{d}{d s} \hat{f}+\frac{\tilde{n}}{2} \hat{f}=K \Theta(1-z) \Theta(2 \pi-\hat{\alpha}) \Theta(\hat{\alpha}) z \delta(s) .
$$




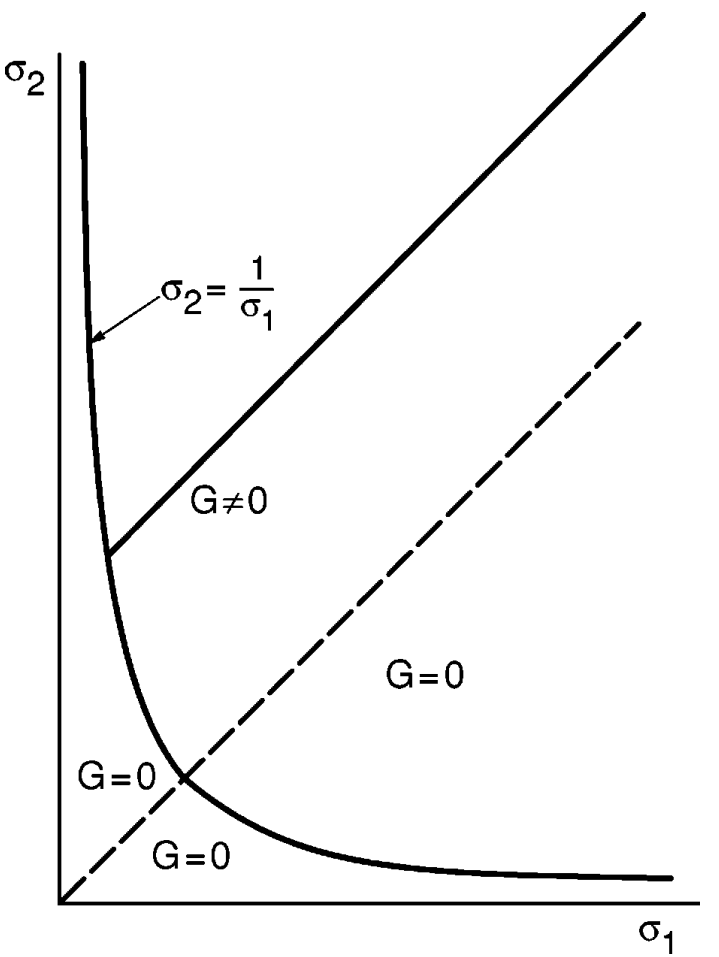

FIG. 3. Solution regions for Eq. (B1) using the method of characteristics. A characteristic line is indicated parallel to the line $\sigma_{1}$ $=\sigma_{2}$.

This equation can now be solved along the characteristic lines in the region indicated in Fig. 3. It has the simple solution

$$
\hat{f}=\frac{\tilde{n}}{2 \pi} \Theta(1-z) \Theta(s) \Theta(\hat{\alpha}) \Theta(2 \pi-\hat{\alpha}) z \exp \left(-\frac{\tilde{n}}{2} s\right) .
$$

When the solution is inserted in the expression for $h_{\mathrm{KS}}$ $=v\langle\operatorname{Tr} \mathbf{I} / \boldsymbol{\rho}\rangle$,

$$
\begin{aligned}
h_{\mathrm{KS}} & =v \int d \rho_{1} d \rho_{2} d \rho_{12} g\left(\rho_{1}, \rho_{2}, \rho_{12}\right)\left(\frac{1}{\rho_{1}}+\frac{1}{\rho_{2}}\right) \\
& =\frac{2 v}{a} \int d s d z d \hat{\alpha} \hat{f}(s, z, \hat{\alpha})\left(\frac{1}{z+s}+\frac{1}{1 / z+s}\right),
\end{aligned}
$$

the results obtained agree with those given by Eq. (28).

The calculation of the maximum Lyapunov exponent starts from the observation that the deviations $\delta \vec{r}_{\perp}(t)$ are given by a product of random matrices acting on the initial deviation $\delta \vec{r}_{\perp}(0)$ [see Eq. (42)]. The random matrix $\mathbf{R}\left(t_{j+1}, t_{j}\right)$ [Eq. (43)] depends on the time of free flight between two collisions and the initial matrix $\rho^{+}$given by $[a(2 \cos \phi)] \mathbf{P}^{-1}$. $\mathbf{P}$ [see Eq. (55)] is parametrized by two collision angles $\phi, \alpha$. Another way of parametrizing this initial matrix is by using its random eigenvalues $z$ : $=\sigma_{1}^{0}=\cos \phi$ and $\sigma_{2}^{0}=1 / z$ and the random angle variable $\alpha$.
Notice that the characteristic lines are identical to the free flight solutions for the eigenvalues of $(2 / a) \boldsymbol{\rho}$ and its offdiagonal element $\sigma_{12}$ [compare Eq. (B3)]. They are chosen such that the initial conditions of the characteristic lines $(s$ $=0)$ are the values of $\sigma_{i}, \sigma_{12}$ immediately after a scattering event with scattering angles $\phi, \alpha$, Therefore, Eq. (B8) can be interpreted as the distribution function for the random variables $z=\cos \phi$ (cosine of the polar scattering angle), $\alpha$ (azimuthal scattering angle), and the time of free flight $s$.

The calculation of the maximum eigenvalue is now a straightforward application of the theory of products of random matrices [24]. It follows from Eq. (42) that the maximum Lyapunov exponent is given by

$$
\lim _{t \rightarrow \infty} \frac{1}{t} \ln |\mathbf{\Pi} \mathbf{R} \delta \vec{r}(0)| /|\delta \vec{r}(0)|,
$$

with $\Pi \mathbf{R}=\prod_{j=0}^{\infty} \mathbf{R}(j, j+1)$. Using an analogous decomposition of the product as in Eq. (11) and the identity $t$ $=\sum_{i=1}^{N} s_{i}$, valid right after the $N$ th collision, with $s_{i}$ the time of free flight between collisions $i-1$ and $i$, Eq. (B10) is equivalent to

$$
\left.\lambda_{\max }=\frac{1}{\langle s\rangle} \overline{\left\langle\ln \left|\mathbf{R}(z, \alpha, s) \cdot \vec{e}_{\psi}\right|\right.}\right\rangle^{\psi} .
$$

Here $\vec{e}_{\psi}$ is a unit vector $\vec{e}_{\psi}=(\cos \psi, \sin \psi)$ and $\overline{\langle\rangle} \psi$ indicates an average over the distribution of $(z, \alpha, s)$ plus an additional average over a stationary distribution $D(\psi)$ of directions $\vec{e}_{\psi}$. This distribution is a solution of a Frobenius-Perron equation

$$
D(\psi)=\int_{0}^{2 \pi} d \psi^{\prime} D\left(\psi^{\prime}\right) \delta\left[\psi^{\prime}-\cos ^{-1}\left(\frac{\vec{e}_{x} \cdot \mathbf{R}(z, \alpha, s) \cdot \vec{e}_{\psi}}{\left|\mathbf{R}(\mathbf{z}, \alpha, s) \cdot \overrightarrow{\mathbf{e}}_{\psi}\right|}\right)\right] .
$$

As we will show shortly, this additional average is not necessary in the equilibrium case since $\psi$ can be absorbed in a redefinition of the azimuthal scattering angle $\alpha$. The equation for $\mathbf{R}$ can be derived from Eq. (43),

$$
\frac{d \mathbf{R}}{d s}=v \boldsymbol{\rho}^{-1} \mathbf{R}
$$

In equilibrium, this is easily solved using $\boldsymbol{\rho}(s)=\boldsymbol{\rho}^{+}+v \mathbf{1} s$,

$$
\mathbf{R}(z, \alpha, s)=\mathbf{1}+\boldsymbol{\rho}^{+-1} s \approx \frac{2 \cos \phi}{a} \mathbf{P} s
$$

The last approximation is valid in the low-density limit since the most important contributions to the average over $s$ come from large time of free flight $s . \mathbf{P}$ is defined in Eq. (55). With this we obtain 


$$
\left|\mathbf{R}(z, \alpha, s) \cdot \vec{e}_{\psi}\right|=\sqrt{\left(\frac{1+z^{2}}{z}\right)^{2}+\left(\frac{1-z^{2}}{z}\right)^{2}+2 \frac{\left(1+z^{2}\right)\left(1-z^{2}\right)}{z^{2}} \cos [2(\alpha-\psi)] s}
$$

From Eq. (B15) it is obvious that $\left|\mathbf{R}(z, \alpha, s) \cdot \vec{e}_{\psi}\right|$ is statistically independent of $\psi$, i.e., the dependence on $\psi$ can be absorbed in a redefinition of $\alpha$. To obtain $\lambda_{\max }$ we have to calculate the average of Eq. (B15) using the distribution function (B8). The result agrees with Eq. (27).

[1] E. H. Hauge, in Transport Phenomena, edited by G. Kirczenow and J. Marrow, Lecture Notes in Physics Vol. 31 (Springer-Verlag, Berlin, 1974), p. 337.

[2] E. G. D. Cohen, Colloq. Int. (CNRS) 236, 269 (1974).

[3] Ya. G. Sinai, Russ. Math. Surv. 25, 137 (1970); L. A. Bunimovich and Ya. G. Sinai, Commun. Math. Phys. 78, 479 (1981); Ya. G. Sinai and N. I. Chernov, Russ. Math. Surv. 42, 181 (1987); L. A. Bunimovich, in Dynamical Systems II, edited by Ya. G. Sinai (Springer-Verlag, Berlin, 1989), p. 151; P. Gaspard, in Quantum Chaos, edited by G. Casati, I. Guarneri, and U. Smilansky (North-Holland, Amsterdam, 1993), p. 307.

[4] Ya. G. Sinai, Funct. Anal. Appl. 13, 192 (1980).

[5] G. Gallavotti and D. S. Ornstein, Commun. Math. Phys. 38, 83 (1974).

[6] N. I. Chernov and C. Haskell, Erg. Th. Dyn. Syst. 16, 19 (1996).

[7] N. S. Krylov, Works on the Foundations of Statistical Physics, edited by Ya. G. Sinai (Princeton University Press, Princeton, 1979).

[8] N. I. Chernov, Funct. Anal. Appl. 25, 204 (1991).

[9] J.-P. Eckmann and D. Ruelle, Rev. Mod. Phys. 57, 617 (1985).

[10] P. Dahlqvist, Nonlinearity 10, 159 (1997); see also P. Dahlqvist, J. Stat. Phys. 84, 773 (1996).

[11] H. van Beijeren and J. R. Dorfman, Phys. Rev. Lett. 74, 4412 (1995).

[12] H. van Beijeren, J. R. Dorfman, E. G. D. Cohen, H. A. Posch, and Ch. Dellago, Phys. Rev. Lett. 77, 1974 (1996).

[13] A. Latz, H. van Beijeren, and J. R. Dorfman, Phys. Rev. Lett. 78, 207 (1997); see also J. R. Dorfman and H. van Beijeren, Physica A 240, 12 (1997).

[14] A. Latz, Z. Phys. B 103, 351 (1997).

[15] P. Gaspard and G. Nicolis, Phys. Rev. Lett. 65, 1693 (1990).

[16] D. J. Evans and G. P. Morriss, Statistical Mechanics of Nonequilibrium Liquids (Academic, London, 1990); W. G. Hoover, Computational Statistical Mechanics (Elsevier, Amsterdam, 1991).

[17] Ch. Dellago and H. A. Posch, Phys. Rev. E 52, 2401 (1995); Phys. Rev. Lett. 78, 211 (1997).

[18] S. Chapman and T. G. Cowling, Mathematical Theory of Non-
Uniform Gases (Cambridge University Press, Cambridge, 1970).

[19] V. I. Arnold, Mathematical Methods of Classical Mechanics (Springer-Verlag, Berlin, 1989).

[20] P. Gaspard and J. R. Dorfman, Phys. Rev. E 52, 3525 (1995).

[21] Ch. Dellago, H. A. Posch, and W. G. Hoover, Phys. Rev. E 53, 1485 (1996).

[22] H. Flanders, Differential Forms with Applications to the Physical Sciences (Dover, New York, 1989).

[23] G. Vattay, Prog. Theor. Phys. Suppl. 116, 251 (1994).

[24] D. Mannion, Ann. Appl. Prob. 3, 1189 (1993); A. Crisanti, G. Paladin, and A. Vulpiani, Products of Random Matrices in Statistical Physics (Springer-Verlag, Berlin, 1993).

[25] J. R. Dorfman and E. G. D. Cohen, J. Math. Phys. 8, 282 (1967).

[26] J. R. Dorfman and H. van Beijeren, in Statistical Mechanics, edited by B. J. Berne (Plenum, New York, 1977), pt. B.

[27] A. Weijland and J. M. J. van Leeuwen, Physica (Amsterdam) 36, 457 (1967); 38, 3 (1968); see also C. Bruin, ibid. 72, 261 (1974).

[28] M. H. Ernst and A. Weijland, Phys. Lett. 34A, 39 (1971).

[29] D. J. Evans, E. G. D. Cohen, and G. P. Morriss, Phys. Rev. A 42, 5990 (1990).

[30] C. P. Dettmann and G. P. Morriss, Phys. Rev. E 53, R5545 (1996).

[31] M. P. Wojtkowski and C. Liverani (unpublished).

[32] E. G. D. Cohen and T. Berlin, Physica (Amsterdam) 26, 717 (1960).

[33] D. Ruelle, Thermodynamic Formalism (Addison-Wesley, Reading, MA, 1978).

[34] C. Beck and F. Schlögl, Thermodynamics of Chaotic Systems (Cambridge University Press, Cambridge, 1993).

[35] C. Appert, H. van Beijeren, M. H. Ernst, and J. R. Dorfman, Phys. Rev. E 54, R1013 (1996); J. Stat. Phys. 87, 1253 (1997).

[36] H. van Beijeren, J. R. Dorfman, Ch. Dellago, and H. A. Posch, Phys. Rev. E 56, 5272 (1997).

[37] R. van Zon and H. van Beijeren, Phys. Rev. Lett. (to be published).

[38] E. Courant and D. Hilbert, Methods of Mathematical Physics (Wiley-Interscience, New York, 1962), Vol. II. 